\title{
A milestone toward understanding PDR properties in the extreme environment of LMC-30 Doradus *
}

\author{
M. Chevance ${ }^{1,2}$, S. C. Madden ${ }^{1}$, V. Lebouteiller ${ }^{1}$, B. Godard ${ }^{3}$, D. Cormier ${ }^{4}$, F. Galliano ${ }^{1}$, S. Hony ${ }^{4}$, R. Indebetouw ${ }^{5,6}$ \\ J. Le Bourlot ${ }^{3}$, M.-Y. Lee ${ }^{1}$, F. Le Petit ${ }^{3}$, E. Pellegrini ${ }^{4}$, E. Roueff ${ }^{3}$, and R. $\mathrm{Wu}^{7}$ \\ ${ }^{1}$ Laboratoire AIM, CEA/DSM - CNRS - Université Paris Diderot, IRFU/Service d'Astrophysique, CEA Saclay, \\ 91191 Gif-sur- Yvette, France \\ e-mail: melanie.chevance@cea.fr \\ 2 Université Paris Diderot, Sorbonne Paris Cité, 75205 Paris, France \\ 3 LERMA, Observatoire de Paris \& CNRS, 5 place Jules Janssen, 92190 Meudon, France \\ ${ }^{4}$ Institut für theoretische Astrophysik, Zentrum für Astronomie der Universität Heidelberg, Albert-Ueberle Str. 2, 69120 Heidelberg, \\ Germany \\ 5 Department of Astronomy, University of Virginia, PO Box 3818, Charlottesville, VA 22903, USA \\ 6 National Radio Astronomical Observatory, Charlottesville, VA 22904, USA \\ 7 Department of Astronomy, Graduate School of Science, The University of Tokyo, Bunkyo-ku, 113-0033 Tokyo, Japan
}

Received 12 November 2015 / Accepted 14 March 2016

\begin{abstract}
Context. More complete knowledge of galaxy evolution requires understanding the process of star formation and the interaction between the interstellar radiation field and interstellar medium (ISM) in galactic environments traversing a wide range of physical parameter space. We focus on the impact of massive star formation on the surrounding low metallicity ISM in 30 Doradus in the Large Magellanic Cloud (LMC). A low metal abundance, which can characterizes some galaxies of the early Universe, results in less ultraviolet (UV) shielding for the formation of the molecular gas necessary for star formation to proceed. The half-solar metallicity gas in this region is strongly irradiated by the super star cluster R136, making it an ideal laboratory to study the structure of the ISM in an extreme environment.

Aims. Our goal is to construct a comprehensive, self-consistent picture of the density, radiation field, and ISM structure in the most active star-forming region in the LMC, 30 Doradus. Our spatially resolved study investigates the gas heating and cooling mechanisms, particularly in the photodissociation regions (PDR) where the chemistry and thermal balance are regulated by far-UV photons $(6 \mathrm{eV}<$ $h v<13.6 \mathrm{eV})$.

Methods. We present Herschel observations of far-infrared (FIR) fine-structure lines obtained with PACS and SPIRE/FTS. We combined atomic fine-structure lines from Herschel and Spitzer observations with ground-based CO data to provide diagnostics on the properties and structure of the gas by modeling it with the Meudon PDR code. For each tracer we estimate the possible contamination from the ionized gas to isolate the PDR component. We derive the spatial distribution of the radiation field, the pressure, the size, and the filling factor of the photodissociated gas and molecular clouds.

Results. We find a range of pressure of $\sim 10^{5}-1.7 \times 10^{6} \mathrm{~cm}^{-3} \mathrm{~K}$ and a range of incident radiation field $G_{\mathrm{UV}} \sim 10^{2}-2.5 \times 10^{4}$ through PDR modeling. Assuming a plane-parallel geometry and a uniform medium, we find a total extinction $A_{V}^{\max }$ of 1-3 mag, which corresponds to a PDR cloud size of 0.2 to $3 \mathrm{pc}$ with small CO depth scale of 0.06 to $0.5 \mathrm{pc}$. At least $90 \%$ of the [C II] originates in PDRs in this region, while a significant fraction of the $L_{\mathrm{FIR}}$ (up to $70 \%$ in some places) can be associated with an ionized gas component. The high $[\mathrm{O} \mathrm{III]}] /[\mathrm{C}$ II] ratio ( 2 to 60 ) throughout the observed map, correlated with the filling factor, reveals the porosity of the ISM in this region, which is traversed by hard UV photons surrounding small PDR clumps. We also determine the three-dimensional structure of the gas, showing that the clouds are distributed 20 to 80 pc away from the main ionizing cluster, R136.
\end{abstract}

Key words. ISM: general - photon-dominated region (PDR) - Magellanic Clouds - ISM: individual objects: LMC-30 Doradus ISM: clouds - ISM: structure

\section{Introduction}

Galaxy evolution is dictated by progressive chemical enrichment, which is mostly achieved through a succession of star formation episodes. The effect of metal enrichment, on what we observe to be the star formation and interstellar medium (ISM) properties, remains elusive despite circumstantial evidence. For example, reduced metallicity is expected to have

\footnotetext{
* The reduced images are only available at the CDS via anonymous ftp to cdsarc.u-strasbg. fr $(130.79 .128 .5)$ or via http://cdsarc.u-strasbg.fr/viz-bin/qcat?J/A+A/590/A36
}

important consequences on the chemistry and the subsequent heating and cooling mechanisms of the gas and dust, directly affecting the transition of the atomic to molecular phase. In low metallicity environments, the transition between $\mathrm{C}^{+} / \mathrm{C} / \mathrm{CO}$ can be shifted further into the cloud in physical scale, leaving a relatively larger photodissociation region (PDR) and a smaller CO core compared to more metal-rich environments, such as the Milky Way (Kaufman et al. 1999). This effect on the molecular cloud structure manifests itself in an observed low CO luminosity in dwarf galaxies (e.g., Cormier et al. 2014; Schruba et al. 2012) and requires a higher $\mathrm{CO}-$ to- $\mathrm{H}_{2}$ conversion factor, i.e., the 
$X_{\mathrm{CO}}$ factor (Schruba et al. 2012; Bolatto et al. 2013). The low observed CO luminosity could also possibly be explained by a higher star formation efficiency.

Dwarf galaxies in our local Universe are the closest environments we can explore in detail to witness the interplay between star formation and ISM under low metallicity conditions. Large surveys probing the cooling of dwarf galaxies are possible for the first time with the Herschel Space Observatory (Pilbratt et al. 2010; e.g., the Dwarf Galaxy Survey, DGS; Madden et al. 2013). Recent studies taking advantage of the Herschel sensitivity, have modeled the dust and gas properties of a wide range of low metallicity galaxies on integrated galaxy scales (e.g., Rémy-Ruyer et al. 2013, 2014, 2015; Cormier et al. 2012, 2015; Cigan et al. 2016) and find prominent differences between metal-rich and metal-poor galaxies. For example, from far-infrared (FIR) line ratios, Cormier et al. (2015) determined that radiation fields over global galaxy scales are harder in starforming dwarf galaxies compared to more metal-rich galaxies. Furthermore, the filling factor of the ionized gas appears larger relatively to the neutral gas. As a consequence of the low metallicity and low extinction in dwarf galaxies, it is possible that a significant fraction of the molecular gas is not traced by $\mathrm{CO}$, but may be residing in the $\mathrm{C}^{+}$or $\mathrm{C}^{0}$ - emitting region; for example (referred to as the "dark gas" in Wolfire et al. 2010), this is quantified in low metallicity environments using $\mathrm{C}^{+}$by Poglitsch et al. (1995) and Madden et al. (1997) and, more recently, in our Galaxy by Langer et al. (2014) and Pineda et al. (2014).

The Large Magellanic Cloud (LMC) is our closest low metallicity galaxy neighbor $\left(1 / 2 Z_{\odot}\right.$; Rolleston et al. 2002; Pagel $2003 ; 50 \mathrm{kpc}$; Walker 2012) allowing us to zoom into the ISM at the spatial resolution of $\sim 12^{\prime \prime}(\sim 3 \mathrm{pc})$ with Herschel. We focus on 30 Doradus (hereafter "30Dor"), which is the most prominent star-forming region in the LMC and provides the best laboratory to study the impact of a super star cluster (SSC) on the ISM. The primary ultraviolet (UV) radiation source illuminating this region is the SSC R136, containing 39 O3 stars (Hunter 1999), which are often considered to be the most extreme starforming region in the Local Group. The lower dust abundance of the LMC allows for deep penetration of the ionizing radiation, creating extended PDR regions and a more porous environment channeling the UV photons. Observations at the Atacama Large (sub)Millimeter Array (ALMA) from Indebetouw et al. (2013) revealed the clumpy structure of the molecular gas in 30Dor, showing small ${ }^{12} \mathrm{CO}$ filaments and clumps $(\lesssim 1 \mathrm{pc})$ covering about $15 \%$ of their map. We examine the PDR conditions in the neutral atomic gas using mainly [C II] and [O I] observed by Herschel in this region to unveil the spatial distribution of the radiation field and structure of the photodissociated gas and molecular clouds. The fraction of CO-dark gas based on this detailed study will be quantified in a following study (Chevance et al., in prep., hereafter Paper II).

Observations of the ionized gas were conducted with Spitzer and studied by Indebetouw et al. (2009). They showed in particular that photoionization dominates the ionization structure of the gas over shocks in the H II region around R136. A study of the fine structure lines of $\mathrm{C}^{+}$and $\mathrm{O}^{0}$ in 30Dor has been previously carried out by Poglitsch et al. (1995) with the Kuiper Airborne Observatory (KAO) at a resolution of $\sim 55^{\prime \prime}(\sim 13 \mathrm{pc})$. These authors found that a highly fragmented structure with high density clouds $\left(n=10^{3}-10^{4} \mathrm{~cm}^{-3}\right)$, of low relative beam filling factor of CO compared to the PDR (4\% of the clumps volume), bathed in ionized gas could explain the observed ratio [C II]/CO (i.e., ten times higher than the Galactic value). Moreover, most of the molecular gas may be present in the PDR and faint in $\mathrm{CO}$. Now, the PACS observations provide better spatial resolution than the KAO data and include other important tracers with an improved signal-to-noise ratio (S/N). Pineda et al. (2012) investigated the $\mathrm{CO}$ and $[\mathrm{CI}]$ emission observed with the NANTEN2 4-m telescope in a 26" beam, combined with the KAO observations of [C II] in 30Dor, and found likewise a very clumpy medium.

We present spectroscopic data of 30Dor in Sect. 2. Section 3 describes the observed maps and some preliminary results. In Sect. 4 we use PDR models to determine the physical parameters of the gas in PDR and we study the impact of metallicity and geometry on these parameters. We discuss our results and build a comprehensive 3D picture of the region in Sect. 5. Key results and conclusions are summarized in Sect. 6.

\section{Observations and data preparation}

\subsection{Herschel PACS spectroscopy}

We have mapped five fine structure lines, [C II] $158 \mu \mathrm{m}$, [N II] $122 \mu \mathrm{m},[\mathrm{OI}$ ] $63 \mu \mathrm{m}$, [O I] $145 \mu \mathrm{m}$, and [O III] $88 \mu \mathrm{m}$ using the Photodetector Array Camera and Spectrometer (PACS; Poglitsch et al. 2010) toward 30Dor. Properties of these lines are presented in Table 1 and the maps can be seen in Fig. 1. These observations, described in Madden et al. (2013), are part of the Herschel key program, SHINING (P.I. E. Sturm). We also used two additional pointings east of R136, which were observed by Indebetouw et al (OT2) in [C II], [O I] $63 \mu \mathrm{m}$, [N II] $122 \mu \mathrm{m}$, and [O III] $88 \mu \mathrm{m}$. The details of the observations are shown in Appendix A.

The PACS array is composed of $5 \times 5$ spatial pixels (or spaxels) of $9.4^{\prime \prime}$ covering a total field of view of $47^{\prime \prime}$. The fine structure lines [O I] $63 \mu \mathrm{m}$, [O III] $88 \mu \mathrm{m}$, [N II] $122 \mu \mathrm{m}$, [O I] $145 \mu \mathrm{m}$, and [C II] were mapped with respectively $25,25,4,11$, and 31 raster positions, covering approximately a $4^{\prime} \times 5^{\prime}$ region $(56 \mathrm{pc} \times 70 \mathrm{pc})$. The observations were carried out in unchopped mode. The beam size is $9.5^{\prime \prime}$ at $60 \mu \mathrm{m}$, and $12^{\prime \prime}$ at $160 \mu \mathrm{m}$ (PACS Observer's Manual 2011).

We refer to Cormier et al. (2015) for the full description of the PACS observations and data reduction, and we summarize some of the main steps here. The data are reduced with the Herschel interactive processing environment (HIPE) v12.0.0 (Ott 2010) from Level 0 to Level 1. The Level 1 cubes, calibrated in flux and wavelength, are then exported and processed with PACSman v3.61 (Lebouteiller et al. 2012) to fit the lines and create the individual maps. Each spectrum is fitted with a second order polynomial for the baseline and a Gaussian for the line. Finally the individual rasters are projected onto a common grid of $3^{\prime \prime} \times 3^{\prime \prime}$ pixels $(0.72 \times 0.72 \mathrm{pc})$ to reconstruct the final maps. Uncertainties on the fit and projection are estimated using a Monte-Carlo simulation. All of the lines are well detected everywhere in the map (Fig. 1). The weakest line, [N II] $122 \mu \mathrm{m}$ has a $\mathrm{S} / \mathrm{N}$ between 5 and 30 for most of the mapped area. The emission line [O I] $145 \mu \mathrm{m}$ has a S/N between 7 and 90 and the $\mathrm{S} / \mathrm{N}$ is above 10 for all of the other lines.

The observed intensities match well those detected with the KAO by Poglitsch et al. (1995) with a lower spatial resolution (55" for [C II] and [OI] $145 \mu \mathrm{m}$ and $22^{\prime \prime}$ for [OI] $63 \mu \mathrm{m})$. For example, they found a maximum [C II] intensity of $10^{-3} \mathrm{erg} \mathrm{s}^{-1} \mathrm{~cm}^{-2} \mathrm{sr}^{-1}$, and we measure a maximum intensity of $1.1 \times 10^{-3} \mathrm{erg} \mathrm{s}^{-1} \mathrm{~cm}^{-2} \mathrm{sr}^{-1}$ on PACS data convolved to the same resolution. They are also similar to the $[\mathrm{C}$ II] intensities measured by Requena-Torres (in prep.) using the German REceiver for 
Table 1. Properties of lines and observations.

\begin{tabular}{|c|c|c|c|c|c|}
\hline Instrument & Transition & $\lambda(\mu \mathrm{m})$ & $F W H M(\operatorname{arcsec})$ & Ionization energy $(\mathrm{eV})$ & $n_{\text {crit }}{ }^{a}\left(\mathrm{~cm}^{-3}\right)$ \\
\hline PACS & $\begin{array}{l}{[\mathrm{O} \text { I }]{ }^{3} P_{1}-{ }^{3} P_{2}} \\
{[\mathrm{O} \mathrm{I}]{ }^{3} P_{0}-{ }^{3} P_{1}} \\
{[\mathrm{O} \text { III }]{ }^{3} P_{1}-{ }^{3} P_{0}} \\
{[\mathrm{C} \mathrm{II}]{ }^{2} P_{3 / 2}-{ }^{2} P_{1 / 2}} \\
{[\mathrm{~N} \mathrm{II}]{ }^{3} P_{2}-{ }^{3} P_{1}}\end{array}$ & $\begin{array}{c}63.2 \\
145.5 \\
88.3 \\
157.7 \\
121.8 \\
\end{array}$ & $\begin{array}{c}9.5 \\
11.0 \\
9.5 \\
11.6 \\
9.9 \\
\end{array}$ & $\begin{array}{c}- \\
- \\
35.1 \\
11.3 \\
14.5 \\
\end{array}$ & $\begin{array}{c}4.7 \times 10^{5}[\mathrm{H}] \\
9.5 \times 10^{4}[\mathrm{H}] \\
510[\mathrm{e}] \\
2.8 \times 10^{3}[\mathrm{H}], 50[\mathrm{e}] \\
310[\mathrm{e}]\end{array}$ \\
\hline SPIRE/FTS & $\begin{array}{l}[\mathrm{N} \mathrm{II}]]^{3} P_{1}-{ }^{3} P_{0} \\
{[\mathrm{CI}]^{3} P_{2}-{ }^{3} P_{1}} \\
{[\mathrm{CI}]{ }^{3} P_{1}-{ }^{3} P_{0}}\end{array}$ & $\begin{array}{l}205.3 \\
370.4 \\
609.7\end{array}$ & $\begin{array}{l}16.6 \\
36.2 \\
38.6 \\
\end{array}$ & $\begin{array}{c}14.5 \\
- \\
-\end{array}$ & $\begin{array}{c}48[\mathrm{e}] \\
1.2 \times 10^{3}\left[\mathrm{H}_{2}\right] \\
4.7 \times 10^{3}\left[\mathrm{H}_{2}\right]\end{array}$ \\
\hline Spitzer/IRS ${ }^{b}$ & 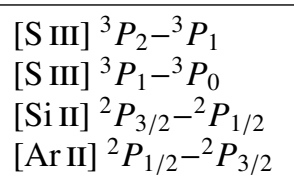 & $\begin{array}{c}18.7 \\
33.5 \\
34.8 \\
7.0\end{array}$ & $\begin{array}{l}4.9 \\
8.9 \\
9.4 \\
2.0\end{array}$ & $\begin{array}{c}23.3 \\
23.3 \\
8.1 \\
15.8\end{array}$ & $\begin{array}{c}2 \times 10^{4}[\mathrm{e}] \\
7 \times 10^{3}[\mathrm{e}] \\
3.4 \times 10^{5}[\mathrm{H}], 1 \times 10^{3}[\mathrm{e}] \\
4.0 \times 10^{5}[\mathrm{e}]\end{array}$ \\
\hline Mopra $^{c}$ & ${ }^{12} \mathrm{CO} J=1 \rightarrow 0$ & 2600 & 43 & - & $1.8 \times 10^{3}\left[\mathrm{H}_{2}\right]$ \\
\hline $\mathrm{ASTE}^{d}$ & ${ }^{12} \mathrm{CO} J=3 \rightarrow 2$ & 867 & 22 & - & $3.2 \times 10^{4}\left[\mathrm{H}_{2}\right]$ \\
\hline
\end{tabular}

Notes. ${ }^{(a)}$ Critical densities are noted [e] for collisions with electrons $(T=10000 \mathrm{~K}),[\mathrm{H}]$ with hydrogen atoms $(T=100 \mathrm{~K})$, and $\left[\mathrm{H}_{2}\right]$ with molecular hydrogen $\left(T=10 \mathrm{~K}\right.$, in the optically thin limit). ${ }^{(b)}$ Indebetouw et al. (2009); ${ }^{(c)}$ Wong et al. (2011); ${ }^{(d)}$ Minamidani et al. (2011).

Astronomy at Terahertz frequencies (GREAT; Heyminck et al. 2012) instrument on the Stratospheric Observatory For Infrared Astronomy (SOFIA).

\subsection{Herschel SPIRE spectroscopy}

The Spectral and Photometric Imaging Receiver (SPIRE) instrument includes an Imaging Fourier Transform Spectrometer (FTS) covering the wavelength ranges 194-324 and 316-672 $\mu \mathrm{m}$ (SPIRE Short Wavelength SSW and SPIRE Long Wavelength SLW arrays, respectively). The 30Dor nebula was observed with the SPIRE FTS in the high spectral resolution $(\Delta v \sim 1.2 \mathrm{GHz})$, intermediate spatial sampling mode. In the intermediate spatial sampling mode, SLW and SSW are moved between four jiggling positions with a spacing of $\sim 28^{\prime \prime}$ and $\sim 16^{\prime \prime}$, respectively. The observations were performed on January 8, 2013 (observation IDs: 1342219550,1342257932 and 1342262908 ) with a total integration time of $\sim 15400 \mathrm{~s}$.

We process the FTS data using HIPE version 11.0.2825 and the SPIRE calibration version 11.0 (Fulton et al. 2010; Swinyard et al. 2013). We use the method from Wu et al. (2013) to derive integrated intensity images and their uncertainties. This script has recently been used to generate FTS spectral cubes for M83 (Wu et al. 2015). A combination of parabola (continuum) and sinc (emission) functions is used to model a spectral line. The spectra are then projected onto a grid that covers a $5^{\prime} \times 5^{\prime}$ area with a pixel size of $15^{\prime \prime}$ (roughly corresponding to the detector spacing for SSW). We perform a Monte-Carlo simulation with 300 iterations to estimate the uncertainties on the spectra, as described in detail in Lee et al. (in prep.). The $\mathrm{S} / \mathrm{N}$ is between 1 and 8 for [N II] $205 \mu \mathrm{m}$ and between 0.5 and 5 for [C I $] 370 \mu \mathrm{m}$. The [C I] $609 \mu \mathrm{m}$ is weaker and the $\mathrm{S} / \mathrm{N}$ is below 2 .

The maps of [N II] $205 \mu \mathrm{m}$ and [C I] 370 and $609 \mu \mathrm{m}$ are presented in Figs. 1 and 2. Properties of these lines are presented in Table 1. The CO transitions from $J=4-3$ to $J=13-12$ were also observed in 30Dor and will be presented in Lee et al. (in prep.).

\subsection{Herschel and Spitzer photometry}

To constrain the PDR models, we need to calculate the infrared luminosity, which requires photometry data from mid-infrared (MIR) to submillimeter. The PACS and SPIRE maps of the LMC at 100, 160250,350 , and $500 \mu \mathrm{m}$ were first published in Meixner et al. (2013) as part of the HERITAGE project. We also use the observations of 30Dor obtained as part of the Spitzer (Werner et al. 2004) Legacy program "Surveying the Agents of a Galaxy's Evolution" (SAGE; Meixner et al. 2006). We used the four channels of the Infrared Array Camera (IRAC; Fazio et al. 2004) at 3.6, 4.5, 5.8, and $8.0 \mu \mathrm{m}$ and the Multiband Imaging Photometer for Spitzer (MIPS; Rieke et al. 2004) observations at 24 and $70 \mu \mathrm{m}$. The MIPS $24 \mu \mathrm{m}$ map is saturated in several pixels. We use the Spitzer Infrared Spectrometer (IRS) spectra (Indebetouw et al. 2009; see also Sect. 2.4) to calculate the $24 \mu \mathrm{m}$ synthetic photometry in the MIPS 24 bandpass and compare to the original map with excellent agreement in areas where the Spitzer/MIPS map is not saturated. Table 2 summarizes the photometry data we use to construct the infrared luminosity associated with their spatial resolution.

\subsection{Spitzer/IRS spectroscopy}

The Spitzer/IRS low-resolution data were initially presented in Indebetouw et al. (2009). The observed lines and their spatial resolution are listed in Table 1 . We reduced again the low spectral resolution cubes with CUBISM (Smith et al. 2007a) as part of an effort to measure lines that have not been investigated yet in detail, in particular, the $\mathrm{H}_{2}$ lines, [Si II], and [Ar II]. The resolving power of both short-wavelength/low-resolution (SL) and long-wavelength/low-resolution (LL) modules range approximately from 60 to 120 (Spitzer Observer's Manual $7.12006^{1}$ ). The maps of [Si II], [S III] $18 \mu \mathrm{m}$, and [S III] $33 \mu \mathrm{m}$ are presented in Fig. 1.

We use the total emission of the polycyclic aromatic hydrocarbon molecules (PAHs), which has been fitted

\footnotetext{
1 Available at http://ssc.spitzer.caltech.edu/documents/
} SOM 

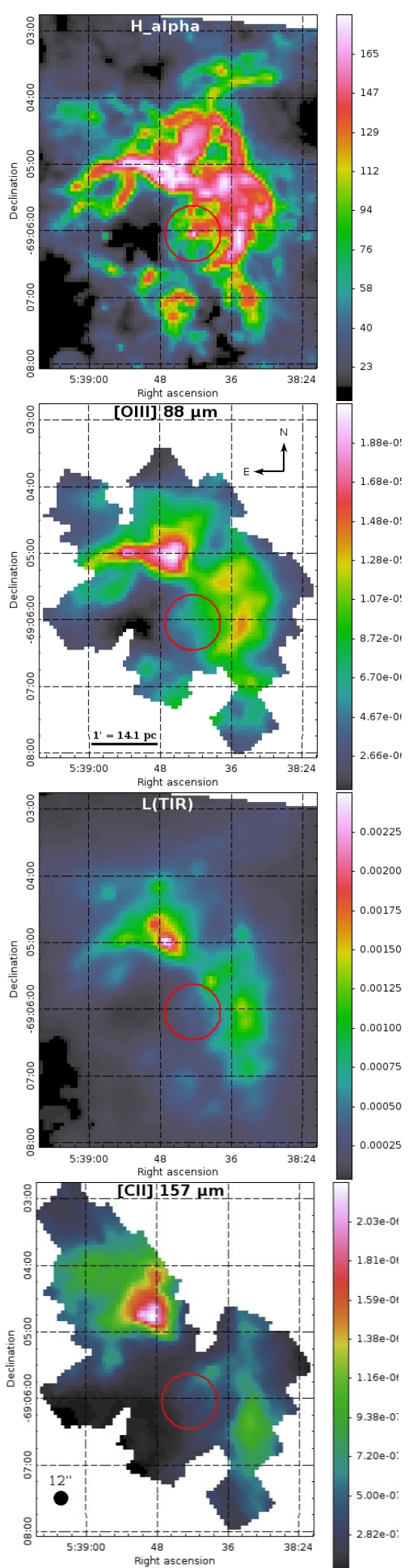
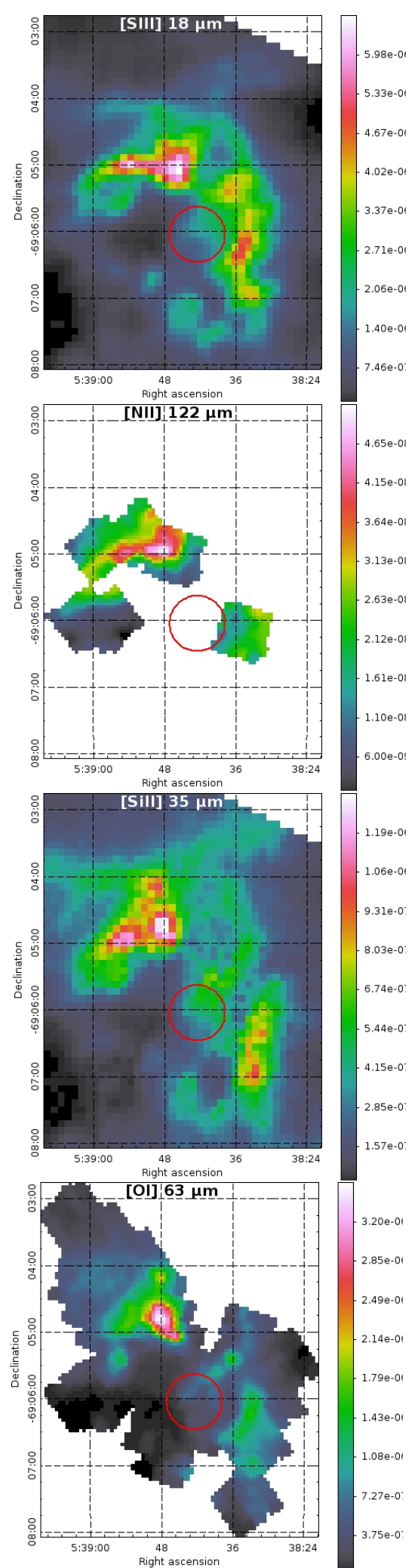
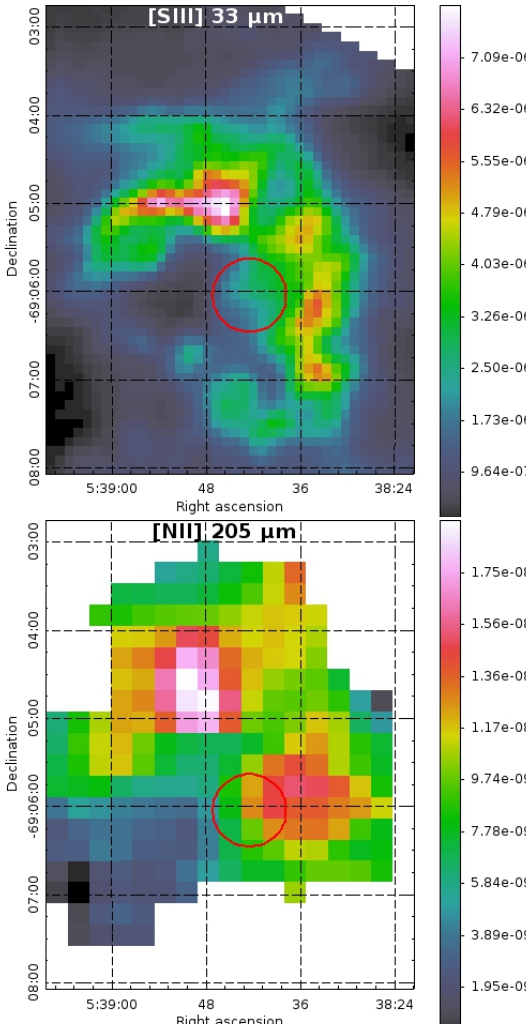

- 5 - -0:
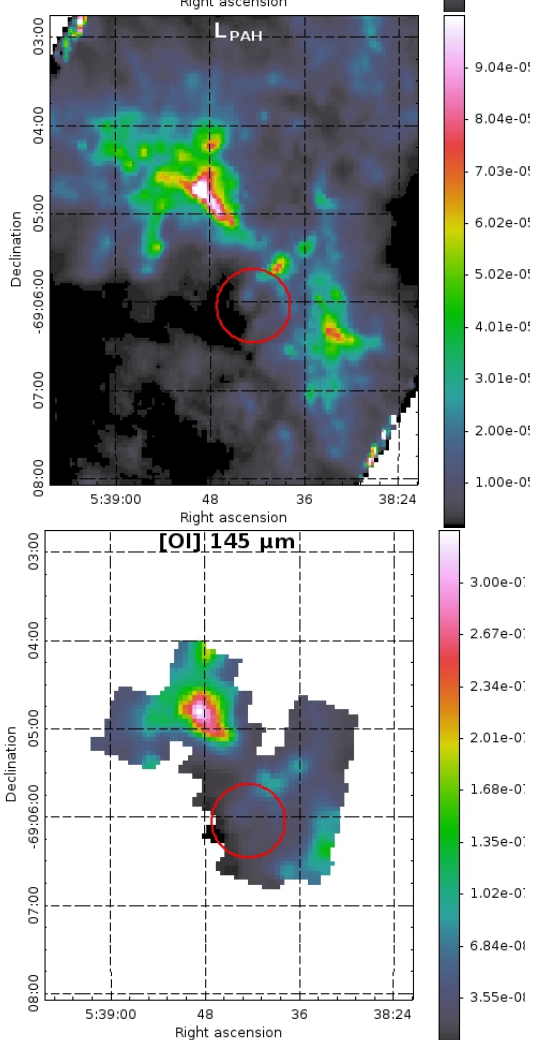

Fig. 1. Maps of PDR and ionized gas tracers from Herschel (PACS and SPIRE/FTS) and Spitzer/IRS observations of the 30Dor region in $\mathrm{W} \mathrm{m}{ }^{-2} \mathrm{sr}^{-1}$. The maps are shown at their original spatial resolution. The $L_{\mathrm{TIR}}$ map is the total infrared luminosity integrated between $3-1000 \mu \mathrm{m}$ from our SED modeling. The red circle represents the location of the R136 cluster. Table 1 and Sect. 2 describe these observations in detail.

by Indebetouw et al. (2009) using the package PAHFIT (Smith et al. 2007b). Finally, we also use the Spitzer/IRS highresolution spectra presented by Lebouteiller et al. (2008) to measure the $\mathrm{H}_{2}$ lines.

\subsection{Ground-based observations}

Low- $J$ CO transitions are required for additional constraints for the PDR modeling. We use the CO $J=1-0$ transition observed 
M. Chevance et al.: A milestone toward understanding PDR properties in the extreme environment of LMC-30 Doradus
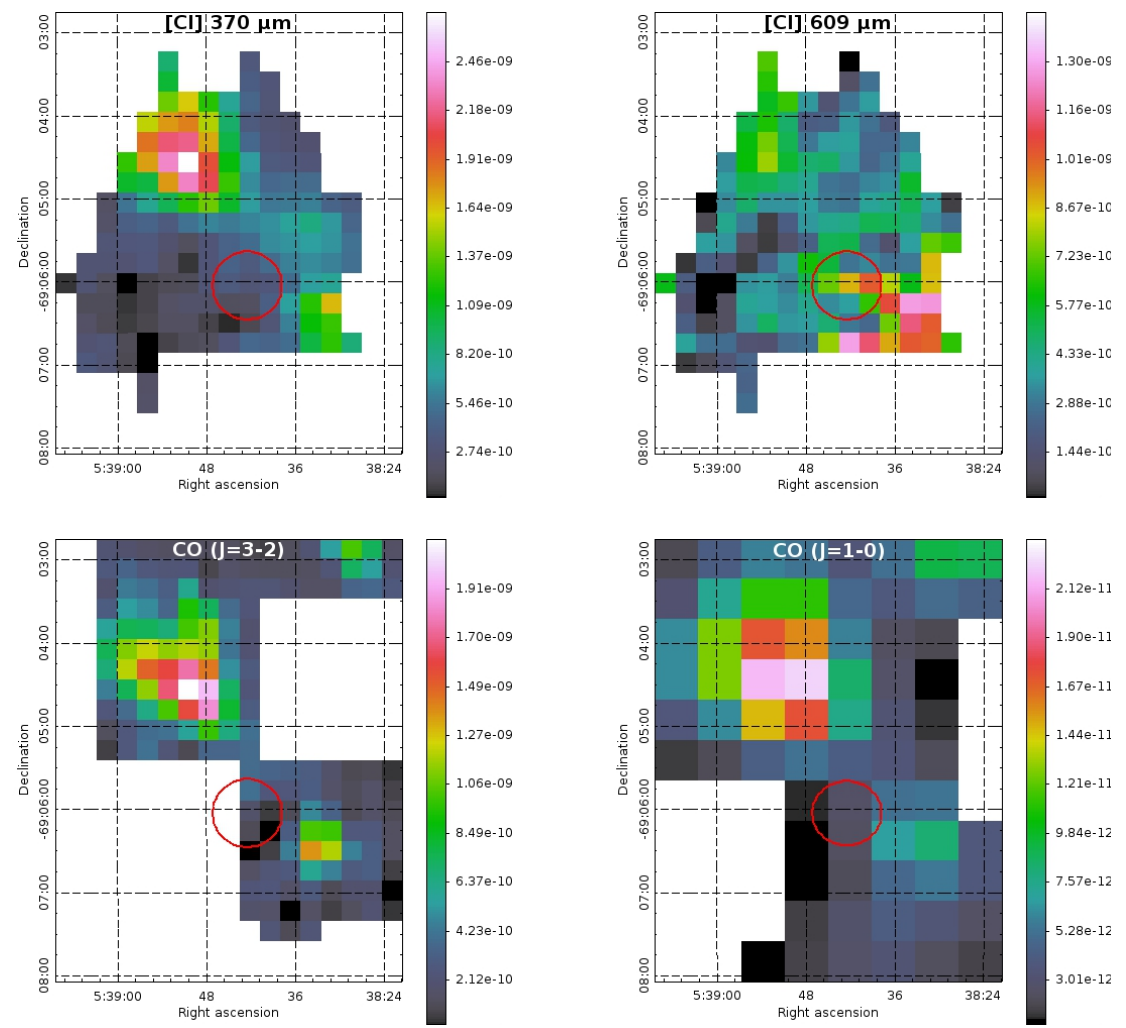

Fig. 2. Top panels: [C I] $370 \mu \mathrm{m}$ (left) and [C I] $609 \mu \mathrm{m}$ (right) SPIRE/FTS maps of 30Dor. Bottom panels: CO (3-2) ASTE map (left) and CO (1-0) Mopra map (right). All of the maps are shown at their original resolution, in $\mathrm{W} \mathrm{m}^{-2} \mathrm{sr}^{-1}$. The circle shows the location of R136. Table 1 and Sect. 2 describe these observations in detail.

Table 2. Properties of the photometry data.

\begin{tabular}{lcc}
\hline \hline Instrument & $\lambda(\mu \mathrm{m})$ & FWHM (arsec) \\
\hline IRAC $^{a}$ & 3.6 & 1.7 \\
& 4.5 & 1.7 \\
& 5.8 & 1.7 \\
& 8.0 & 1.9 \\
\hline MIPS & 24 & 6 \\
& 70 & 18 \\
\hline PACS $^{b}$ & 100 & 7.7 \\
& 160 & 12 \\
\hline SPIRE $^{b}$ & 250 & 18 \\
& 350 & 25 \\
& 500 & 37 \\
\hline
\end{tabular}

References. (a) SAGE (Meixner et al. 2006); (b) HERITAGE (Meixner et al. 2013)

with Mopra (Wong et al. 2011) and CO $J=3-2$ observed with the Atacama Submillimeter Telescope Experiment (ASTE; Minamidani et al. 2011; see Fig. 2). The spatial resolutions for the Mopra and ASTE data are 42" and 22", respectively.

The $\mathrm{H}_{\alpha}$ emission, which we use as a qualitative tracer of the ionized gas, was observed with the Cerro Tololo Inter-American Observatory (CTIO) Curtis Schmidt telescope as part of the Magellanic Clouds Survey (MCELS; priv. comm.; R. Leiton) at a resolution of $\sim 5^{\prime \prime}$.

\subsection{Convolution kernels}

As we use line ratios of different wavelengths and different instruments (see Table 1), we must first convolve the maps to the same resolution. We add quadratically $12 \%$ uncertainties to the PACS maps to account for the absolute calibration uncertainties (PACS Observer's Manual v2.5.1). When we use only PACS observations, all of the maps are convolved to the resolution of PACS at $160 \mu \mathrm{m}\left(12^{\prime \prime}\right.$ or $\left.\sim 3 \mathrm{pc}\right)$, using the kernels from Aniano et al. (2011).

When we combine PACS, SPIRE, and ground-based spectroscopy data together to include the $[\mathrm{CI}]$ and $\mathrm{CO}$ lines (see Sect. 4.4), all of the maps are smoothed to match the resolution of $42^{\prime \prime}(\sim 10 \mathrm{pc})$ limited by the SPIRE long wavelength data. For this, we use the appropriate kernels to convolve a PACS point spread function (modeled by Aniano et al. 2011) to the SPIRE/FTS beam profile (fitted by a two-dimensional HermiteGaussian function) at the lowest resolution, essentially following the method by Gordon et al. (2008).

The photometry bands are used to determine the infrared luminosity with our spectral energy distribution (SED) model (see Sect. 2.7). Since we wish to perform the PDR analysis on the smallest possible scale (i.e., limited by the point spread function of the PACS [C II] map), we calculate the infrared luminosity at the resolution of $12^{\prime \prime}$, which is also the resolution of the PACS $160 \mu \mathrm{m}$ band. We compared this approximated infrared luminosity to that determined using all available bands, i.e., including the SPIRE bands $(250,300$ and $500 \mu \mathrm{m})$. We find little difference on the integrated luminosity per surface area. Thus we include only the bands between 24 and $160 \mu \mathrm{m}$ to fit the SED at the best spatial resolution possible.

\subsection{Infrared luminosity maps}

For each pixel of the map, we construct the full MIR to submm SED, to which we apply the dust SED model of Galliano et al. (2011, AC composition). This is a phenomenological SED fitting 
procedure with which we derive a resolved total infrared luminosity $\left(L_{\mathrm{TIR}}\right)$ between 3 and $1000 \mu \mathrm{m}$ and a FIR luminosity $\left(L_{\mathrm{FIR}}\right)$ between 60 and $200 \mu \mathrm{m}$. This model was designed to fit the Herschel broadband photometry of the LMC, still remaining consistent with the elemental abundances. The free parameters include the dust mass (from which the extinction magnitude in $V$ band, $A_{V}^{\text {dust }}$, can be derived), minimum starlight intensity, difference between the maximum and minimum starlight intensities, starlight intensity distribution power-law index, and PAHto-total dust mass ratio $f_{\mathrm{PAH}}$. The final map of $L_{\mathrm{TIR}}$, which integrates the SED fit between 3 and $1000 \mu \mathrm{m}$, can be seen in Fig. 1. Contrary to other dust parameters, the infrared luminosity is very marginally model dependent. It depends mainly on the wavelength coverage of the photometric constraints used, which in our case is sufficient. For the PDR modeling (see Sect. 4) we use the $L_{\text {FIR }}$ integrated between 60 and $200 \mu \mathrm{m}$. The SED model is better constrained in this limited wavelength range compared to the fit that includes longer wavelengths so this leads to fewer uncertainties on $L_{\mathrm{FIR}}$. However, even with no constraint longward of $160 \mu \mathrm{m}$, the $L_{\mathrm{TIR}}$ of 30 Dor is still relatively well constrained, as the dust in this region is sufficiently warm to peak at much shorter wavelengths.

\section{Data analysis}

\subsection{General morphology}

Figure 1 shows the maps of $\mathrm{H}_{\alpha}$, [O III], [S III], [N II], [C II], [Si II], and [O I] lines at their initial resolution. We also show the $L_{\mathrm{TIR}}$ (integrated between 3 and $1000 \mu \mathrm{m}$ ) and the PAH emission $\left(L_{\mathrm{PAH}}\right)$. The emission of all lines is distributed in the northern and southern lobes around R136. The emission of all lines peaks near the same location within 5 pc toward the northern lobe with some inhomogeneous emission in the south. The spatial distributions of the $[\mathrm{CI}], \mathrm{CO}(1-0)$, and $\mathrm{CO}(3-2)$ emission are presented in Fig. 2. They show two lobes of emission as well, and the peaks of both the [C I] and $\mathrm{CO}$ emission are shifted about $20^{\prime \prime}$ north of the peak of [C II].

The [C II], [OI], and $L_{\mathrm{PAH}}$ follow each other approximately throughout the map. The [O I] $63 \mu \mathrm{m}$, [O I] $145 \mu \mathrm{m}$, and [C II] emission lines, as well as $L_{\mathrm{PAH}}$, are PDR tracers, although there may be a diffuse component as well, which could be contributing to the emission of [C II] (see Sect. 3.2).

The distributions of the $\mathrm{H}_{\alpha}$, [O III] $88 \mu \mathrm{m}$, [S III], and [N II] lines have a structure that is different from the neutral PDR tracers [C II] and [O I]. The spatial distributions of [O III], [S III], and [N II] follow well the distribution of the ionized gas traced by $\mathrm{H}_{\alpha}$ emission and show, in particular, a characteristic arm-like structure in the northeast of R136. The ionization potentials of $\mathrm{O}^{++}$and $\mathrm{S}^{++}$are $35 \mathrm{eV}$ and $23.3 \mathrm{eV}$ (see Table 1), respectively, so [O III] $88 \mu \mathrm{m}$ and both [S III] $18 \mu \mathrm{m}$ and [S III] $33 \mu \mathrm{m}$ probe the highly ionized gas. The peak of [O III] is shifted from that of $[\mathrm{C}$ II] toward the south, in the direction of R136. The peak of [N II] $122 \mu \mathrm{m}$ is located between the peaks of [O III] $88 \mu \mathrm{m}$ and $[\mathrm{OI}]$, as expected from the values of the ionization potential of each species. [N II] traces the low density and low-excitation ionized gas (critical density for collision with electrons are $\sim 310 \mathrm{~cm}^{-3}$ and $\sim 48 \mathrm{~cm}^{-3}$ for [N II] $122 \mu \mathrm{m}$ and [N II] $205 \mu \mathrm{m}$, respectively).

The distributions of [Si II] $35 \mu \mathrm{m}$ and $L_{\mathrm{TIR}}$ emissions share properties both with the ionized gas ([O III] and [S III]) and the PDR tracers ([O I] and [C II]). The [Si II $] 35 \mu \mathrm{m}$ line and the $L_{\mathrm{TIR}}$ can in principle be used as a PDR tracer, but we also find in

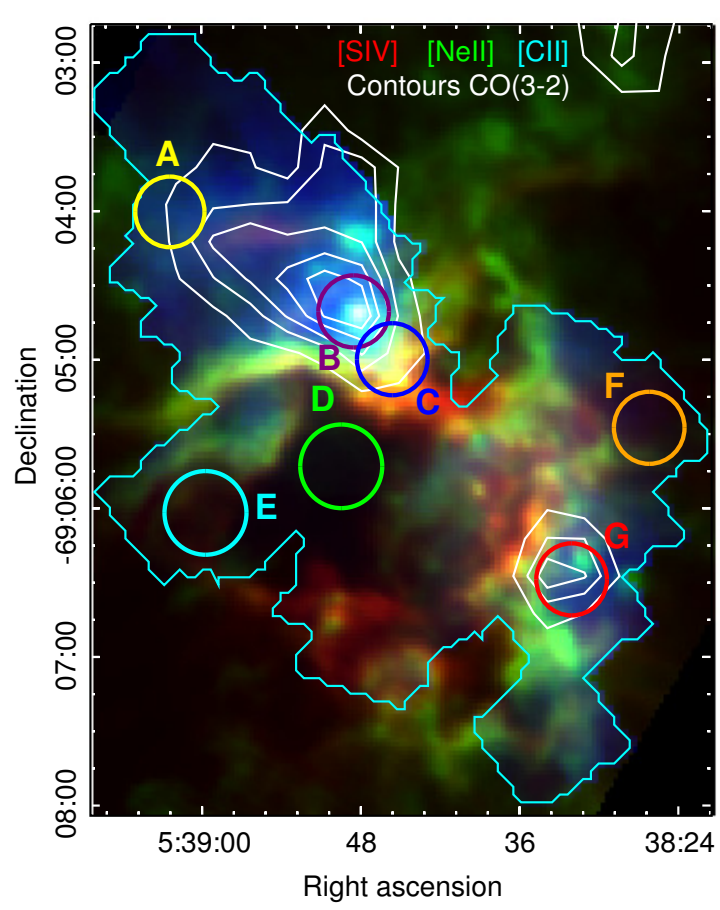

Fig. 3. Red: Spitzer/IRS [S IV] $10.5 \mu \mathrm{m}$ (Indebetouw et al. 2009). Green: Spitzer/IRS [Ne II] $12.8 \mu \mathrm{m}$ (Indebetouw et al. 2009). Blue: PACS [C II] $158 \mu \mathrm{m}$. The blue contours outline the limit of the [C II] map. We can identify the different layers of the gas from the highly ionized medium near the star cluster R136 to the clumpy molecular gas. The contours of the ${ }^{12} \mathrm{CO}(3-2)$ emission observed with ASTE (Minamidani et al. 2011) are indicated in white. The yellow region (A) is located on the northeast of R136, where [C II] is extended but where [O III] seems fainter. The violet circle (B) is located on the [C II] peak, and the blue circle (C) is on the [O III] peak. The green (D), cyan (E), and orange $(\mathrm{F})$ circles probe more diffuse regions. The southwest peak of $[\mathrm{C} \mathrm{II}]$ is in the red region $(\mathrm{G})$.

the maps some features that seem spatially associated with the ionized gas as well. This is discussed in Sects. 3.2 and 3.4.

Figure 3 shows the different layers of the ISM, from the ionization front near the stellar cluster, where the highly ionized gas traced by the [S IV] $10.5 \mu \mathrm{m}$ emission is located, to lower ionization states ([Ne II] $15.6 \mu \mathrm{m})$ and then to the PDRs traced by [C II]. The CO peak is located close to the [C II] peak. This spatial disposition suggests that R136 dominates the photoionization. We can also see that the northern region seems to be shielded very well from ionizing UV photons, since [C II] is very extended in this direction while the tracers of the ionized gas show a sharp decrease on the other side of the $\mathrm{H}_{\alpha}$ arc.

The [O III] $88 \mu \mathrm{m}$ is the brightest FIR line in 30Dor, as in N11, the second largest $\mathrm{H}$ II region of the LMC (Lebouteiller et al. 2012), the dwarf galaxy Haro 11 (Cormier et al. 2012), and in most of the dwarf galaxies (Cormier et al. 2015), integrated over full galaxy scales. This was first noted in several dwarf irregular galaxies in Hunter et al. (2001). [O III] is brighter than [C II] by a factor of 2 to 60 throughout our 30Dor map. Figure 4 shows the ratio [O III $] / L_{\mathrm{TIR}}$ versus $[\mathrm{C} \mathrm{II}] / L_{\mathrm{TIR}}$ in 30Dor. Cormier et al. (2015) already noted the elevated [O III] $88 \mu \mathrm{m} / L_{\mathrm{TIR}}$ in dwarf galaxies compared to the normal galaxies of Brauher et al. (2008). Given that it requires $35 \mathrm{eV}$ to ionize $\mathrm{O}^{+}$to $\mathrm{O}^{++}$, this suggests the presence of high temperature stars throughout the region. The range of $[\mathrm{C} \mathrm{II}] / L_{\mathrm{TIR}}$ values covered in 30 Dor is very broad (more than an order of magnitude) and they cover almost the entire 
M. Chevance et al.: A milestone toward understanding PDR properties in the extreme environment of LMC-30 Doradus

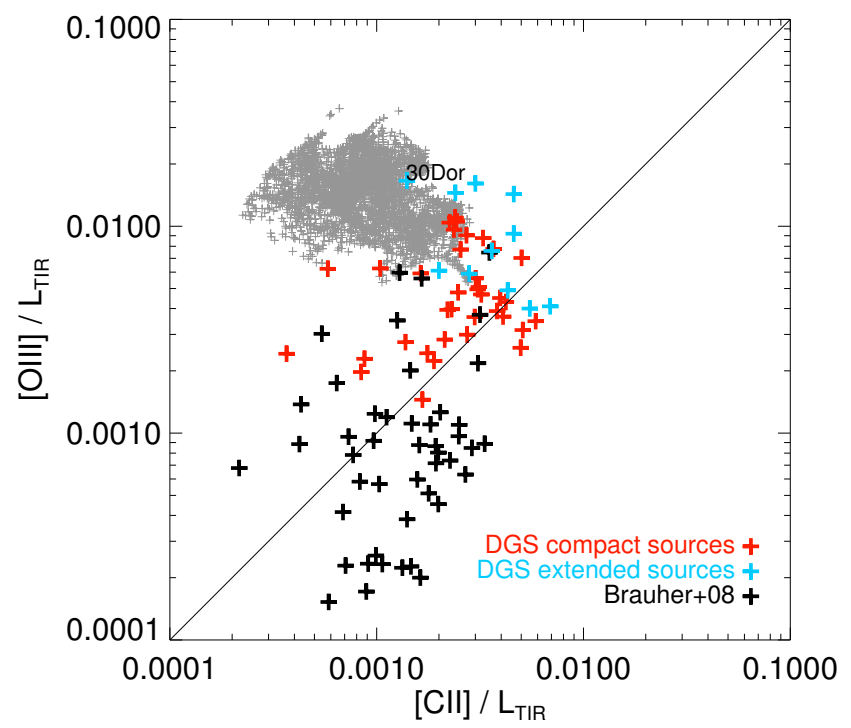

Fig. 4. $[\mathrm{O}$ III $] / L_{\mathrm{TIR}}$ versus $[\mathrm{C} \mathrm{II}] / L_{\mathrm{TIR}}$. The regions in $30 \mathrm{Dor}$ form the gray cloud with the high $[\mathrm{O}$ III $] / L_{\mathrm{TIR}}$ values. The DGS galaxies (Cormier et al 2015) are represented by red (compact) and cyan (extended) symbols. The (mostly) metal-rich galaxies from Brauher et al. (2008) are represented by black symbols.

range of $[\mathrm{C} \mathrm{II}] / L_{\mathrm{TIR}}$ observed in the wide range of galaxy type in the Brauher et al. sample. The $[\mathrm{O} \mathrm{III}] / L_{\mathrm{TIR}}$ distribution is narrower (about a factor of 6 over the map). The regions with the highest $L_{\mathrm{TIR}}$ are the peaks of [O III] and [C II] (Fig. 3; regions C and $\mathrm{B}$ ). The highest [C II] $/ L_{\mathrm{TIR}}$ ratio is found in the northern part of 30Dor (near region A); this is because $L_{\mathrm{TIR}}$ decreases more rapidly than $[\mathrm{C} \mathrm{II}]$ with increasing distance from the exciting sources.

In the regions where [C II] is the brightest, the line intensities of [O I] $63 \mu \mathrm{m}$ and [Si II] are similar to the [C II] intensity. [O I] $63 \mu \mathrm{m}$ is at least ten times brighter than [O I] $145 \mu \mathrm{m}$ everywhere. The map of [N II] $122 \mu \mathrm{m}$ is the smallest and this line is also the faintest of our PACS lines (at least 200 times fainter than $[\mathrm{O} I I I]$ ), but the $\mathrm{S} / \mathrm{N}$ throughout the region mapped is $\geq 5$. The [N II] $205 \mu \mathrm{m}$ emission is 1 to 3 times fainter than [N II] $122 \mu \mathrm{m}$.

\subsection{Origin of $\mathrm{C} / \mathrm{I}$ and Si II emission}

Because the ionization potential of $\mathrm{C}^{0}, 11.3 \mathrm{eV}$, is lower than $13.6 \mathrm{eV}$, the $[\mathrm{CII}]$ line can originate either from the PDRs or from the ionized gas. We thus need to investigate the possible contribution from the ionized gas and from PDRs to the [C II] emission before we can use it as a PDR tracer and a constraint for PDR modeling.

Following the analysis of Oberst et al. (2011), we use the fact that the ratio $[\mathrm{C} \mathrm{II}] /[\mathrm{N}$ II $] 122 \mu \mathrm{m}$ can be calculated theoretically in the ionized gas, and that $[\mathrm{N}$ II] originates only from the ionized gas. We calculate the fine-structure level populations of $\mathrm{C}^{+}$ and $\mathrm{N}^{+}$as a function of the density using the theoretical collisional rates. We then apply a correction factor due to the ionic abundance fraction $\frac{\mathrm{C}^{+} / \mathrm{C}}{\mathrm{N}^{+} / \mathrm{N}}$. We used the MAPPINGS III photoionization grids (Allen et al. 2008) and found that this fraction depends little on the conditions (ionization parameter, starburst age, density) with a value around $0.85 \pm 0.15$. Finally, we scale the emission ratio with the observed elemental abundances of $\mathrm{C}$

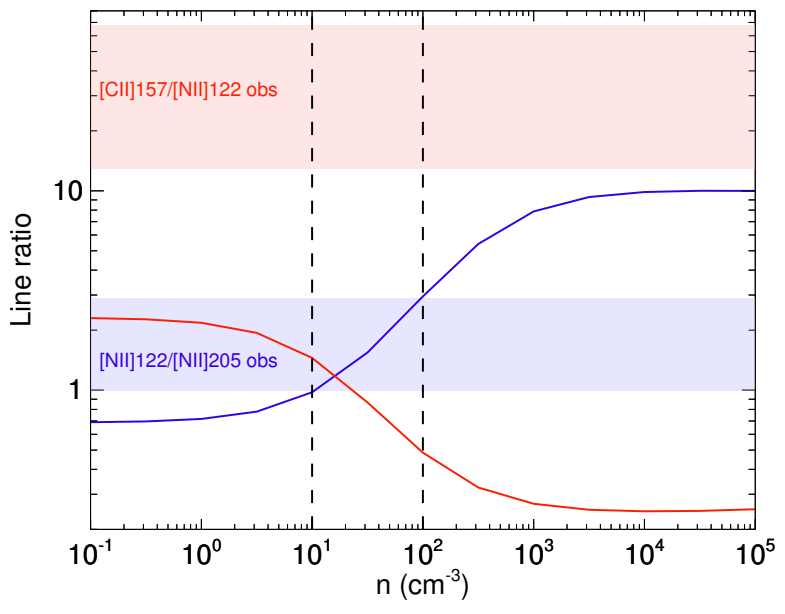

Fig. 5. Theoretical ratios [N II] $122 \mu \mathrm{m} /[\mathrm{N}$ II $] 205 \mu \mathrm{m}$ (blue) and $[\mathrm{C} \mathrm{II}] /[\mathrm{N} \mathrm{II}] 122 \mu \mathrm{m}$ (red) at the temperature of $10000 \mathrm{~K}$. Blue and red areas indicate our observed ranges for [N II] $122 \mu \mathrm{m} /[\mathrm{N} \mathrm{II}] 205 \mu \mathrm{m}$ and [C II $] /[\mathrm{N}$ II $] 122 \mu \mathrm{m}$ ratios, respectively. The observed values of the $[\mathrm{C}$ II $] /[\mathrm{N}$ II $]$ ratio (in red) are much higher than the theoretical value in the ionized gas for the entire map, indicating that $[\mathrm{C} \mathrm{II}]$ is mostly emitted in the PDRs and not in the ionized gas.

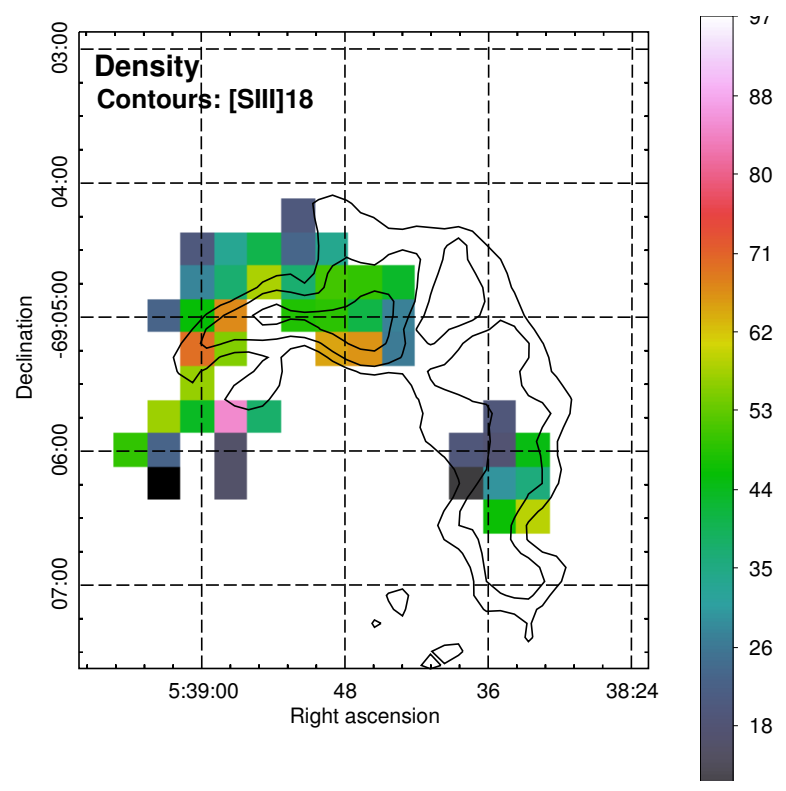

Fig. 6. Electron density in $\mathrm{cm}^{-3}$ calculated from the ratio [N II] $122 \mu \mathrm{m} /\left[\mathrm{N}\right.$ II] $205 \mu \mathrm{m}$. The pixel size is $20^{\prime \prime}$ ( $\left.\sim 5 \mathrm{pc}\right)$. The black contours represent the emission of the ionized gas tracer [S III] $18 \mu \mathrm{m}$.

and $\mathrm{N}$ in 30Dor from Pellegrini et al. (2011) listed in Table 3. The final ratio [C II $] /[\mathrm{N}$ II $] 122 \mu \mathrm{m}$ depends strongly on the electron density between 1 and $1000 \mathrm{~cm}^{-3}$. Since the critical densities for [N II] $122 \mu \mathrm{m}$ and [N II] $205 \mu \mathrm{m}$ are $310 \mathrm{~cm}^{-3}$ and $50 \mathrm{~cm}^{-3}$, respectively (Table 1 ), the ratio [N II] $122 \mu \mathrm{m} /[\mathrm{N} \mathrm{II}]$ $205 \mu \mathrm{m}$ is a good density tracer for the relatively low density ionized gas phase. We calculate the ratio [N II] $122 \mu \mathrm{m} /[\mathrm{N}$ II] $205 \mu \mathrm{m}$ to determine the density using the theoretical curve from Bernard-Salas et al. (2012, see Fig. 5). This ratio depends only slightly on the temperature; we choose a typical temperature of $10000 \mathrm{~K}$. The calculated density presented in Fig. 6 ranges from 10 to $100 \mathrm{~cm}^{-3}$. Our values fall in the low density regime of the $[\mathrm{S} \mathrm{III]}]$ line ratio, which is sensitive to high density $\left(n_{\text {crit }}=1.5 \times 10^{4} \mathrm{~cm}^{-3}\right.$ for $[\mathrm{S} \mathrm{III}] 18 \mu \mathrm{m}$ and $n_{\text {crit }}=4.1 \times 10^{3} \mathrm{~cm}^{-3}$ for [S III] $33 \mu \mathrm{m}$ ), and does not provide a useful constraint. 


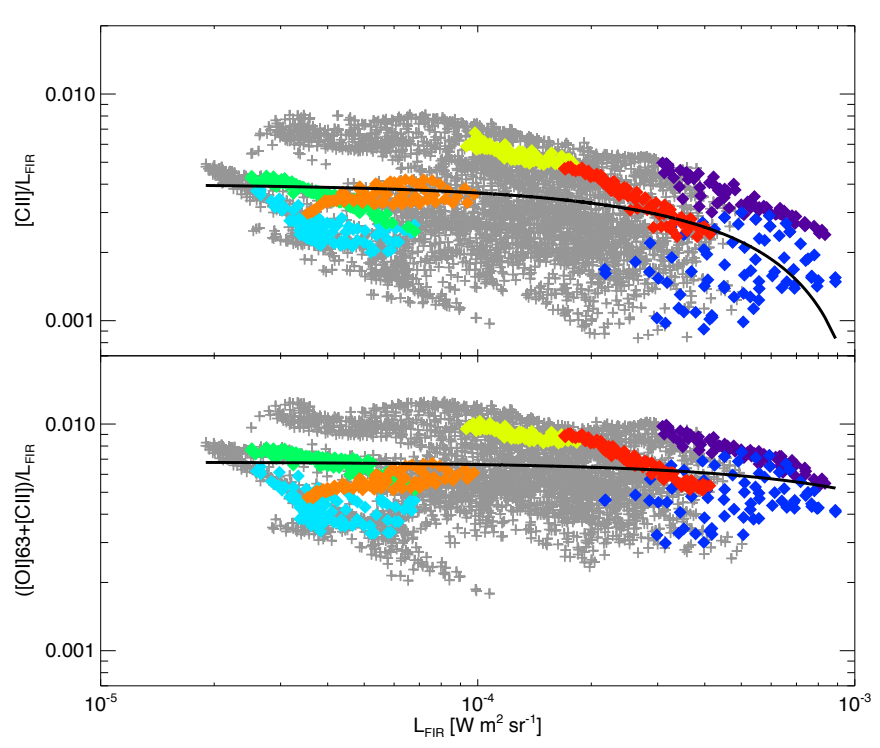

Fig. 7. Top: ratio of [C II $] / L_{\mathrm{FIR}}$ versus $L_{\mathrm{FIR}}$. The color symbols are associated with the regions defined in Fig. 3. The gray symbols are all of the other pixels of our 30Dor map. The black curve shows a linear regression of the data. Bottom: ratio of ([O I $63 \mu \mathrm{m}+[\mathrm{C}$ II $]) / L_{\mathrm{FIR}}$ versus $L_{\mathrm{FIR}}$.

If the [C II] emission originated in the low density ionized gas traced by $[\mathrm{N} \mathrm{II}]$, the $[\mathrm{C} \mathrm{II}] /[\mathrm{N}$ II $] 122 \mu \mathrm{m}$ ratio would be $\sim 0.5-$ 1.3 (Fig. 5). However, the observed ratio is significantly higher by a factor of $\sim 10$ than this theoretical ratio in the ionized gas. At least $90 \%$ of the [C II] is expected to be emitted from PDRs in the entire mapped region. The [C II] emission can be considered to be a reliable tracer of the PDR gas in 30Dor.

Since the density is known and [Ar II] originates from the ionized gas, we can also calculate the theoretical ratio $[\mathrm{C} \mathrm{II}] /[\mathrm{Ar}$ II $]$ in the ionized gas. Similarly, comparing the observed ratio $[\mathrm{C} \mathrm{II}] /[\mathrm{Ar} \mathrm{II}]$ to the theoretical ratio in the ionized gas, we also deduce that a large fraction $(>95 \%)$ of the $[\mathrm{C} \mathrm{II}]$ emission originates from the PDRs.

[Si II] emission can also originate from PDR or ionized gas. We proceed with the same method to separate the emission of the ionized gas from that of the neutral gas. The critical density of [Si II] is close to that of [Ar II] $\left(3.4 \times 10^{5} \mathrm{~cm}^{-3}\right.$ and $4.0 \times$ $10^{5} \mathrm{~cm}^{-3}$, respectively; see Table 1) and both the observed ratios of [Si II]/[Ar II] and [Si II $] /[\mathrm{N} \mathrm{II}]$ are consistent with $60 \%$ to $90 \%$ of the [Si II] emission originating from the PDRs.

\subsection{The photoelectric heating efficiency}

The top panel of Fig. 7 shows the observed $[\mathrm{C} \mathrm{II}] / L_{\mathrm{FIR}}$ ratio as a function of $L_{\text {FIR }}$ for every pixel of the PACS map. The ratio $[\mathrm{C} \mathrm{II}] / L_{\mathrm{FIR}}$ is often used to estimate the fraction of energy absorbed by dust that is used to heat the gas via the photoelectric effect (the photoelectric heating efficiency). This ratio ranges between $0.1 \%$ and $1 \%$ with a significant scatter, i.e., about one order of magnitude. We observe a tendency of decreasing [C II] $/ L_{\text {FIR }}$ as $L_{\text {FIR }}$ increases (same trend as in Stacey et al. 2010 ) with a slope of $-3.6 \pm 0.2$. However, when we add the [O I] $63 \mu \mathrm{m}$ emission (lower panel of Fig. 7), we find a smaller dispersion (with a factor of 7) and also a flatter relation between ([O I $] 63 \mu \mathrm{m}+[\mathrm{C} \mathrm{II}]) / L_{\mathrm{FIR}}$ and $L_{\mathrm{FIR}}$ with a slope of $-1.4 \pm 0.3$ for the linear regression. Both [O I] and [C II] are contributing noticeably to the cooling of the gas, as shown in Lebouteiller et al. (2012).

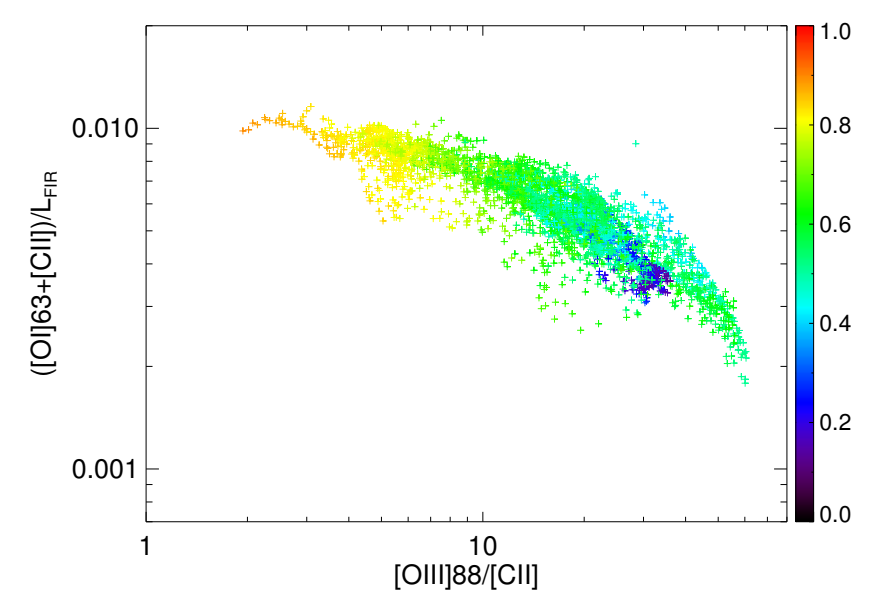

Fig. 8. Ratio of ([O I $63 \mu \mathrm{m}+[\mathrm{C} \mathrm{II}]) / L_{\mathrm{FIR}}$ versus [O III]/[C II]. The color bar indicates the fraction of $L_{\mathrm{FIR}}$ expected to come from the PDRs (see Sect. 3.4).

Figure 8 shows that the decrease of the ratio ([O I] $63 \mu \mathrm{m}+[\mathrm{C}$ II $]) / L_{\mathrm{FIR}}$ is mostly due to the ionized component in $L_{\mathrm{FIR}}$. Indeed, the ratio $([\mathrm{OI}] 63 \mu \mathrm{m}+[\mathrm{CII}]) / L_{\mathrm{FIR}}$ is strongly correlated with the ratio [O III] $88 \mu \mathrm{m} /[\mathrm{CII}]$, and it decreases with increasing [O III] $88 \mu \mathrm{m} /[\mathrm{C} \mathrm{II}]$, which is representative of the ionization state of the gas. If we subtract the infrared contribution from the ionized gas (as described in Sect. 3.4), we find that the ratio $([\mathrm{OI}] 63 \mu \mathrm{m}+[\mathrm{C} \mathrm{II}]) / L_{\mathrm{FIR}}$ is fairly constant and narrow.

\subsection{The origin of the FIR emission}

Although grains and PAHs in the PDRs contribute to the FIR emission, a fraction of $L_{\text {FIR }}$ can also come from the H II regions, i.e., from big grains in equilibrium with the interstellar radiation field (ISRF). In this section, we inspect the origin of the $L_{\mathrm{FIR}}$ throughout the map to separate the fraction in the ionized gas component from that in the PDR component. We assume that the PAH emission traces the PDRs and [O III] traces the ionized phase. Indeed, we can see in Fig. 1 that the spatial distribution of the $L_{\mathrm{FIR}}$ shows features similar to the PAH emission or any other neutral atomic gas tracer ([C II], [O I]), while other features seem to be spatially associated with [O III] or any other ionized gas tracers $\left(\mathrm{H}_{\alpha},[\mathrm{S} \mathrm{III}]\right)$. To disentangle the fraction of $L_{\mathrm{FIR}}$ in the ionized gas, we assume a linear relation such as $L_{\mathrm{FIR}}=\alpha \times L_{\mathrm{PAH}}+\beta \times L_{[\mathrm{OIII}]}$. The pair of $(\alpha ; \beta)$ values is calculated using a multiple linear regression using all of the pixels in the map and is equal to $(5.4 ; 11.5)$. This decomposition implicitly assumes that the PAH-to-dust mass fraction is constant in PDRs and zero in H II regions. The left panel of Fig. 9 presents the correlation between [O III] $88 \mu \mathrm{m} / L_{\mathrm{FIR}}$ and $L_{\mathrm{PAH}} / L_{\mathrm{FIR}}$. The solid line on this plot shows the linear relation defined by $\frac{L_{[\mathrm{O} \text { III }]}}{L_{\mathrm{FIR}}}=\frac{1}{\beta}-\frac{\alpha}{\beta} \times \frac{L_{\mathrm{PAH}}}{L_{\mathrm{FIR}}}$. With this method, we are seeking a first order correction of the total $L_{\mathrm{FIR}}$ to be able to use it for the PDR modeling. The modeled $L_{\text {FIR }}$ reproduces the observed $L_{\text {FIR }}$ within $30 \%$ on average. We determine the proportion of $L_{\mathrm{FIR}}$ coming from the PDRs as $L_{\mathrm{FIR}}^{\mathrm{PDR}}=\alpha L_{\mathrm{PAH}}$. The result is presented on the right panel of Fig. 9. On the north side of the map, far from the ionizing cluster, up to $90 \%$ of the FIR emission is expected to come from the PDRs, while on the east of the map, near the R136 cluster, about $70 \%$ of the FIR emission is expected to come from the ionized gas. We subtract the estimated fraction of $L_{\text {FIR }}$ emitted in the ionized gas, using $L_{\mathrm{FIR}}^{\mathrm{PDR}}$ for the PDR modeling. 
M. Chevance et al.: A milestone toward understanding PDR properties in the extreme environment of LMC-30 Doradus
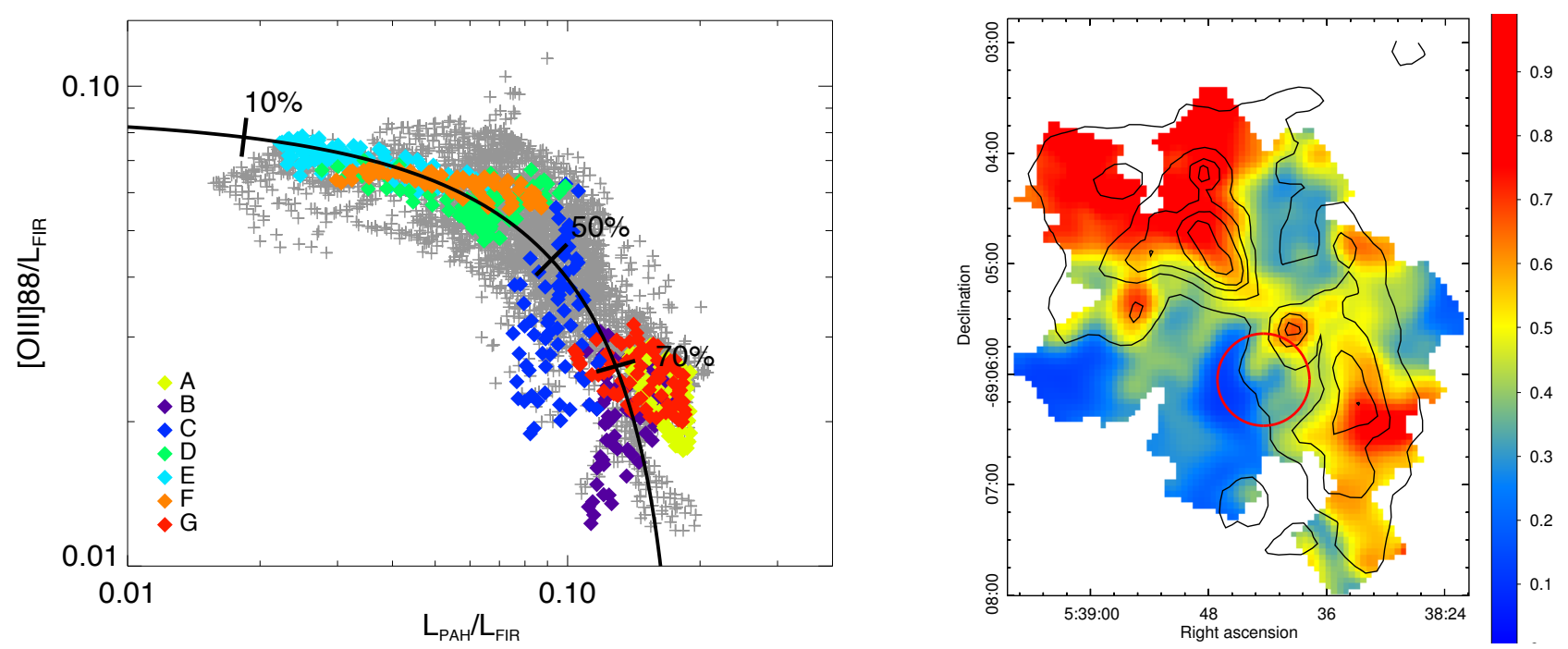

Fig. 9. Left: ratio [O III] $88 \mu \mathrm{m} / L_{\mathrm{FIR}}$ as a function of $L_{\mathrm{PAH}} / L_{\mathrm{FIR}}$. The color symbols are associated with the regions defined in Fig. 3. The gray symbols denote all of the other pixels of our 30Dor map. The solid curve shows the result of the multiple linear regression, using the values $\alpha=5.4$ and $\beta=11.5$. The values indicate the fraction of $L_{\mathrm{FIR}}$ coming from the PDR for three different positions on the curve. Right: ratio of $L_{\mathrm{FIR}}^{\mathrm{PDR}} / L_{\mathrm{FIR}}$. Black contours represent the total FIR emission. Close to the location of R136, only 20 to $30 \%$ of the $L_{\mathrm{FIR}}$ come from the PDRs.

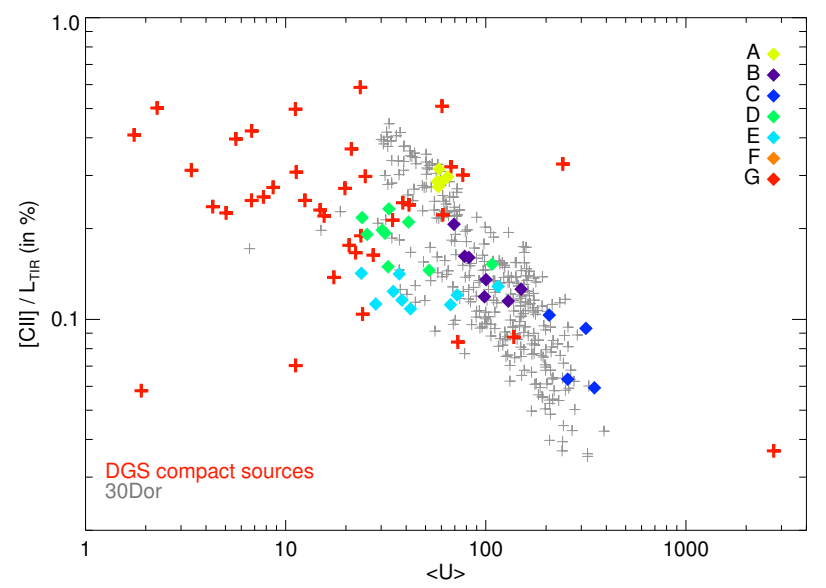

Fig. 10. Correlation between $[\mathrm{C}$ II $] / L_{\mathrm{TIR}}$ and $\langle U\rangle$ (average of the starlight intensity distribution). The red symbols are for the DGS galaxies (Cormier et al. 2015). The colored diamonds correspond to the regions of 30Dor illustrated in Fig. 3.

\subsection{Line ratios: Empirical correlations}

Far-infrared line ratios are useful diagnostics of the ISM conditions. We use these for PDR modeling (Sect. 4) and we inspect their distribution throughout 30Dor. In Figs. 10 and 11, we focus on different regions of 30Dor to inspect the local variations.

To study how the distribution of starlight affects the observed photoelectric heating efficiency, we compare the average modeled starlight $\langle U\rangle$ from the SED modeling (see Sect. 3.4) with [C II] $/ L_{\text {TIR }}$ (Fig. 10). We use the total $L_{\text {TIR }}$ here since we do not know the fraction of the PDR component for the DGS sources. We compare our spatially resolved values with the distribution of $\langle U\rangle$ and $[\mathrm{C}$ II $] / L_{\mathrm{TIR}}$ from the integrated DGS compact sources of Cormier et al. (2015) and Rémy-Ruyer et al. (2014). More details and references can be found in Cormier et al. (2015). We see that 30 Dor covers a large range in $[\mathrm{C} \mathrm{II}] / L_{\mathrm{TIR}}$ of approximately one order of magnitude and approximately one order of magnitude in $\langle U\rangle$.

There is a trend of decreasing [C II] $/ L_{\mathrm{TIR}}$ as $\langle U\rangle$ is increasing, following the trend observed by Cormier et al. (2015), showing

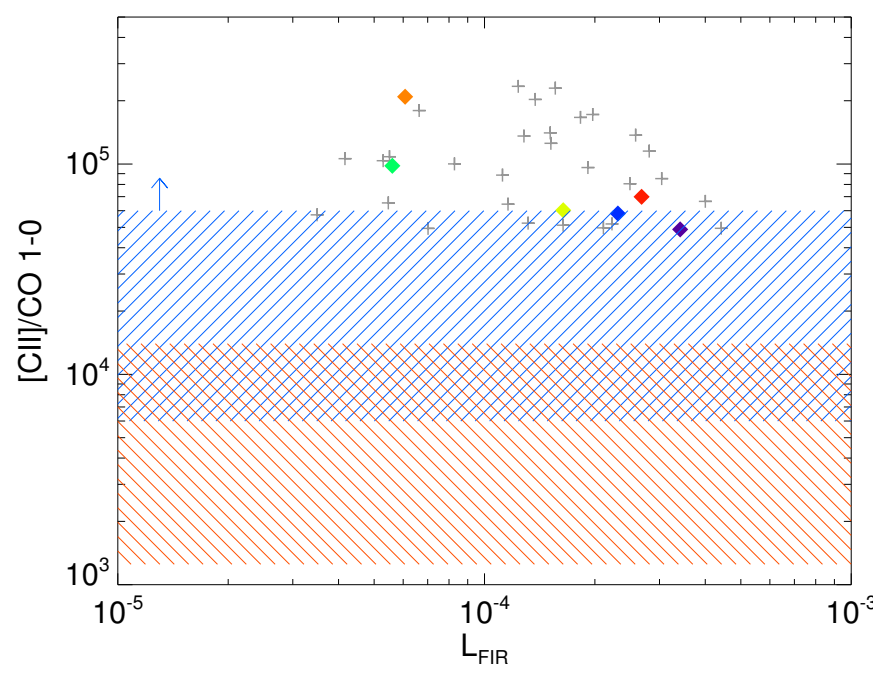

Fig. 11. Ratio of $[\mathrm{C}$ II $] / C O(1-0)$ versus $L_{\mathrm{FIR}}$ at the scale of $30^{\prime \prime}$. The dashed horizontal orange band indicates the observed ratio [C II $] / \mathrm{CO}(1-$ 0 ) in star-forming regions of the Galaxy and metal-rich galaxies (Stacey et al. 1991). The dashed horizontal blue band indicates the range of observed ratios for six bright subsolar metallicity galaxies of the DGS (Cormier et al. 2014).

an apparent line deficit at high $\langle U\rangle$ values. This is probably an effect of the contribution of the ionized component to the infrared luminosity, as shown in Fig. 8. The [O III] peak (region $\mathrm{C}$ in Fig. 3) is the region with the highest $\langle U\rangle$ : this is the closest region to R136 in physical distance (see Sect. 5.3); the gas is mostly ionized. This region also corresponds to the lowest $[\mathrm{C} \mathrm{II}] / L_{\mathrm{TIR}}$ ratio.

To compare one of the primary PDR coolants with the $\mathrm{CO}(1-$ 0 ), we show in Fig. 11 [C II]/CO versus $L_{\text {FIR }}$ at the resolution of $42^{\prime \prime}$. [C II] $/ \mathrm{CO}(1-0)$ throughout 30Dor is $\sim 5 \times 10^{4}-3 \times 10^{5}$. The observed range of the ratio $[\mathrm{C} \mathrm{II}] / \mathrm{CO}(1-0)$ is very broad. These values are about a factor 10 higher than the typical values for spiral or starburst galaxies ( 2000-8000; Stacey et al. 1991; Negishi et al. 2001), and more in agreement with the 
range of values measured for integrated dwarf galaxies as already noticed in Poglitsch et al. (1995), Madden et al. (1997), and Cormier et al. (2010, 2014). We note that low metallicity galaxies always show extreme $[\mathrm{C} \mathrm{II}] / \mathrm{CO}$ compared to the more metal-rich galaxies.

\section{PDR modeling}

In this section, we model the infrared observations of 30Dor using the Meudon PDR code and present results describing the properties of the gas.

\subsection{The Meudon PDR model}

The Meudon PDR code $^{2}$ is described in Le Petit et al. (2006), Le Bourlot et al. (2012), and Bron et al. (2014). It computes the atomic and molecular structure of interstellar clouds. The model considers a 1D stationary plane-parallel slab of gas and dust illuminated by a radiation field (from UV to radio) arising from one or both sides. The radiative transfer is solved in an iterative way at each point of the cloud by taking into account absorption by gas and dust and scattering and emission by dust. For the present work, we used a development version (v1.6.0) with updates that includes the computation of X-ray radiative transfer and the impact on the chemistry and thermal balance of the cloud (Godard et al., in prep.).

\subsection{Input and output parameters}

We describe here some of the configuration parameters of the model. We assume that the gas in each pixel can be modeled by a single cloud of pressure $P$, illuminated by a radiation field. The standard radiation field used in the Meudon PDR code is that observed in the solar neighborhood (Mathis et al. 1983) and is scaled with the parameter $G_{\mathrm{UV}}$ to control the intensity of the incident radiation field on each side of the cloud. For $G_{\mathrm{UV}}=1$, the integrated energy density between $911.8 \AA$ to $2400 \AA$ is $6.8 \times 10^{-14} \mathrm{erg} \mathrm{cm}^{-3}$. In the model we ran, $G_{\mathrm{UV}}$ ranges between 1 and $10^{5}$ on one side and is fixed to 1 on the other side to expose this side to the general interstellar field. The pressure ranges between $10^{4}$ and $10^{8} \mathrm{~cm}^{-3} \mathrm{~K}$. The pressure is constant throughout the cloud, however, we also explored a constant density model (Sect. 4.5). We investigated the possibility of adding X-rays in the model, but found that they are not needed to explain the PACS observed data (Sect. 5.5). The visual extinction of the entire cloud is varied from $A_{V}^{\max }=1$ magnitude to $A_{V}^{\max }=10$ magnitude. We estimate the mass fraction of PAHs from our SED modeling (Sect. 2.7). We find that $f_{\mathrm{PAH}}=1 \%$ is adapted for 30Dor. We use elemental abundances as in Pellegrini et al. (2011) for $\mathrm{He}, \mathrm{C}, \mathrm{N}, \mathrm{O}, \mathrm{Ne}, \mathrm{Si}$, and $\mathrm{S}$ to reproduce the conditions in 30Dor as accurately as possible (see Table 3). The dust-to-gas mass ratio is fixed to $0.5 \times 10^{-3}$ based on our SED modeling (Sect. 2.7).

The output quantities computed by the model include the integrated line intensities and $L_{\mathrm{FIR}}$, ionic and molecular abundances, emissivities, and chemical and thermal structure of the cloud. An example of the variations of the local gas phase abundances for some elements as a function of the depth into the cloud is presented in Fig. 12 for a typical cloud of $A_{V}^{\max }$ of 10 with a constant pressure of $P=10^{6} \mathrm{~cm}^{-3} \mathrm{~K}$, which is illuminated by a radiation field of $G_{\mathrm{UV}}=3000$.

\footnotetext{
2 The Meudon PDR code is public and available online at the following address: http://ism.obspm.fr
}

Table 3. Input parameters for the PDR model.

\begin{tabular}{llc}
\hline \hline Parameter & Notation & Value \\
\hline Pressure & $P$ & $10^{4}-10^{8} \mathrm{~cm}^{-3} \mathrm{~K}$ \\
Radiation field & $G_{\mathrm{UV}}$ & $1-10^{5}$ \\
Cosmic ray flux & $\zeta^{a}$ & $3 \times 10^{-16} \mathrm{~s}^{-1}$ \\
Total visual extinction & $A_{V}^{\max }$ & $1-10 \mathrm{mag}$ \\
Metallicity & $Z^{b}$ & $0.5 Z_{\odot}$ \\
PAH fraction & $f_{\text {PAH }}$ & $1 \%$ \\
Dust-to-gas mass ratio & $M_{\text {dust }} / M_{\text {gas }}$ & $5 \times 10^{-3}$ \\
\hline Gas phase abundances & & $\log (n(\mathrm{X}) / n(\mathrm{H}))$ \\
\hline $\mathrm{He}$ & & -1.05 \\
$\mathrm{C}$ & & -4.3 \\
$\mathrm{~N}$ & & -4.91 \\
$\mathrm{O}$ & & -3.75 \\
$\mathrm{Ne}$ & & -4.36 \\
$\mathrm{Si}$ & & -5.51 \\
$\mathrm{~S}$ & & -5.32 \\
\hline
\end{tabular}

Notes. ${ }^{(a)}$ Indriolo \& McCall (2012), Indriolo et al. (2015); ${ }^{(b)}$ Metallicity: $12+\log (\mathrm{O} / \mathrm{H})=8.38$ (Rolleston et al. 2002; Pagel 2003) and $(\mathrm{O} / \mathrm{H})_{\odot}=4.9 \times 10^{-4}$ (Asplund et al. 2009); ${ }^{(c)}$ Pellegrini et al. (2011).

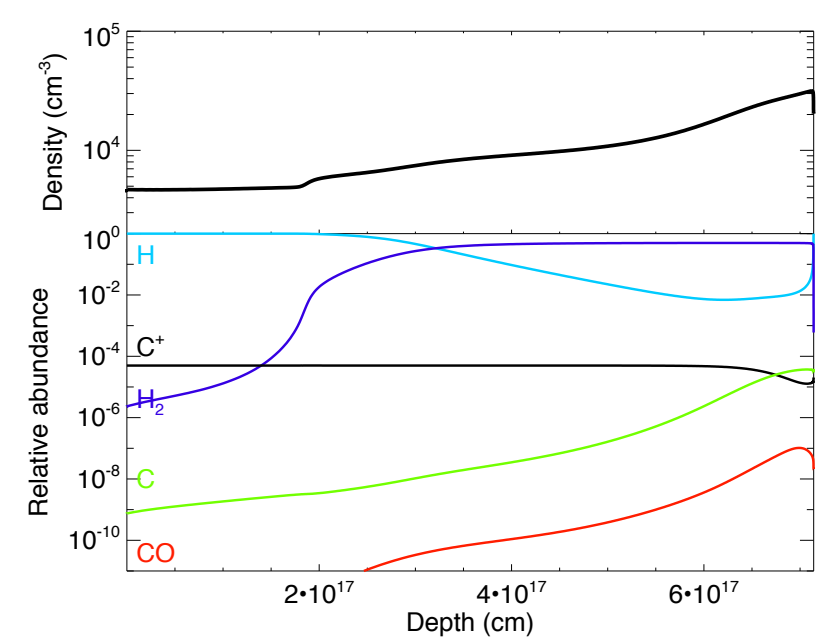

Fig. 12. Top: density profile as a function of the depth into the cloud in a simulated cloud of $A_{V}^{\max }=2, P=10^{6} \mathrm{~cm}^{-3} \mathrm{~K}$ with $G_{\mathrm{UV}}=3000$ on the left side and $G_{\mathrm{UV}}=1$ on the right side. Bottom: local gas phase relative abundances of $\mathrm{C}^{+}, \mathrm{C}, \mathrm{CO}, \mathrm{O}$, and $\mathrm{H}_{2}$ for the same model.

We use [O I], [C II] and $L_{\mathrm{FIR}}^{\mathrm{PDR}}$ to constrain $P$ and $G_{\mathrm{UV}}$ in the PDR model pixel by pixel. We consider here the observed [C II] emission without correction since the contribution from the ionized gas is low (Sect. 3.2) and we correct the $L_{\mathrm{FIR}}$ emission to remove the contamination from the component associated with the ionized gas (see Sects. 3.2 and 3.4). At first we let $A_{V}^{\max }$ vary freely as this set of tracers cannot constrain this parameter and it has no influence on the resulting best model. This is illustrated in Fig. 13, where we show the ratios $\mathcal{R}$ between several modeled line ratios and their observations for simulated clouds of different total depths. The ratios $[\mathrm{OI}] /[\mathrm{C}$ II $],([\mathrm{O} \mathrm{I}]+[\mathrm{C} \mathrm{II}]) / L_{\mathrm{FIR}}$ are not very sensitive to $A_{V}^{\max }$, as is also the case for the ratio [C I] $609 \mu \mathrm{m} /[\mathrm{C} \mathrm{I}] 370 \mu \mathrm{m}$. 
M. Chevance et al.: A milestone toward understanding PDR properties in the extreme environment of LMC-30 Doradus

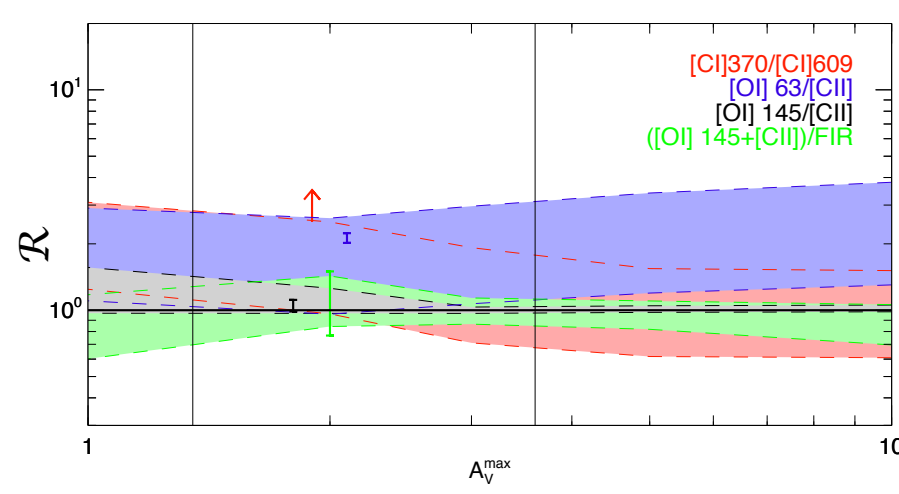

Fig. 13. Ratios, $\mathcal{R}$, of the modeled line ratios over the observed line ratios for [CI] $370 \mu \mathrm{m} /[\mathrm{CI}] 609 \mu \mathrm{m}$, [O I] $63 \mu \mathrm{m} /[\mathrm{C} \mathrm{II}]$, [O I $] 145 \mu \mathrm{m} /[\mathrm{C} \mathrm{II}]$, and $([\mathrm{OI}] 145 \mu \mathrm{m}+[\mathrm{C} \mathrm{II}]) / L_{\mathrm{FIR}}^{\mathrm{PDR}}$, for simulated clouds of different $A_{V}^{\max }$ from 1 to 10 for 30Dor abundances. The width of the bands represents the dispersion of $\mathcal{R}$ throughout the map. The error bars due to uncertainties on the observations are plotted. The vertical lines indicate the range of $A_{V}^{\max }$ where the predictions of the model are compatible with the observed [C II $] / \mathrm{CO}(3-2)$ (see Fig. 17).

\subsection{Pressure and incident radiation field}

We find the best solution for the incident radiation field and the pressure using the observed line intensities as constraints. They are found by minimizing the $\chi^{2}$ distribution

$\chi^{2}=\sum_{j=1}^{N}\left(\frac{I_{j}(x, y)-M_{j}}{\sigma_{j}(x, y)}\right)^{2}$,

where $I_{j}(x, y)$ is the observed value of the ratio $i$ for a given pixel $(\mathrm{x}, \mathrm{y}), \sigma_{j}(x, y)$ is the uncertainty associated with this observed ratio, and $M_{j}$ is the value predicted by the model for the ratio $j$. The value $N$ is the number of constraints (independent ratios) that are used. Ratios of line intensities are used instead of absolute values. In this case, if species are cospatial in the cloud and there are no opacity effects, we can then ignore the effects of an area filling factor that differs from 1.0 and the presence of several clouds along the line of sight (see Sect. 5.1).

As an example, we present in Fig. 14 the values of $G_{\mathrm{UV}}$ and $P$ that reproduce the observed values for the ratios ([O I $] 145 \mu \mathrm{m}+[\mathrm{C} \mathrm{II}]) / L_{\mathrm{FIR}}^{\mathrm{PDR}}$ in blue, [O I] $145 \mu \mathrm{m} /[\mathrm{C} \mathrm{II}]$ in red, and [O I] $63 \mu \mathrm{m} /[\mathrm{C} \mathrm{II}]$ in cyan for two pixels of the map of 30Dor at $12^{\prime \prime}$ resolution, located in the regions $\mathrm{D}$ and $\mathrm{C}$ (Fig. 3). We can see in these figures that the constraint given by the ratio [O I] $63 \mu \mathrm{m} /[\mathrm{C} \mathrm{II}]$ is never consistent with the other ratios within the error bars. This is likely due to optical depth effects in the [O I] $63 \mu \mathrm{m}$ line, as we show later in this section. For this reason, we do not consider this line to be one of the constraints to the model.

First, we use the ratios ([O I $] 145 \mu \mathrm{m}+[\mathrm{C}$ II $]) / L_{\mathrm{FIR}}^{\mathrm{PDR}}$ and [O I] $145 \mu \mathrm{m} /[\mathrm{C}$ II $]$ to constrain $G_{\mathrm{UV}}$ and $P$. Thus we are limited by the PACS resolution of $12^{\prime \prime}$ and by the spatial coverage of the [OI] $145 \mu \mathrm{m}$ map. We do not use the ratio [CI] $370 \mu \mathrm{m} /[\mathrm{CI}] 609 \mu \mathrm{m}$, which does not bring strong constraints on $G_{\mathrm{Uv}}$ and $P$ as the error bars are very large. We have then the same number of constraints and parameters. We can note from Fig. 14 that there is a degeneracy between a high $G_{\mathrm{UV}} /$ low $P$ solution and a low $G_{\mathrm{Uv}} /$ high $P$ solution. The addition of the ratio $[\mathrm{C} \mathrm{II}] /[\mathrm{CI}]$ or $[\mathrm{C} \mathrm{II}] / \mathrm{CO}$ does not help to break this degeneracy since they are very dependent on $A_{V}^{\max }$ (Sect. 4.4). However, the high $G_{\mathrm{Uv}} /$ low $P$ solution, highlighted with a green cross in Fig. 14, has a lower $\chi^{2}$ and is preferred based on the following arguments. Indeed, the high $P$ solution requires high optical depths in [O I] $63 \mu \mathrm{m}$ ([O I] $63 \mu \mathrm{m}$ is overpredicted by a factor of up to 9 , see below), while the $A_{V}^{\max }$ (determined as in Sect. 4.4) would be very low ( $<1 \mathrm{mag})$. In addition, we found a pressure of $P=10^{5}-10^{6} \mathrm{~cm}^{-3} \mathrm{~K}$ for a typical temperature of $10000 \mathrm{~K}$ (see Sect. 3.2) in the ionized gas. We find a similar pressure in the PDR, suggesting that the gas may well be in pressure equilibrium. However, it must also be noted that Pellegrini et al. (2011) find a pressure that is somewhat larger using optical lines. Finally, a low $G_{\mathrm{UV}}$ solution results in large physical distances between the clouds and R136 (see Sect. 5.3), which is significantly larger (by a factor of $\sim 10$ to 100) than the distances found in Pellegrini et al. (2011).

We find $G_{\mathrm{UV}}$ ranging between $10^{2}$ and $3 \times 10^{4}$ throughout the region and $P$ between $10^{5} \mathrm{~cm}^{-3} \mathrm{~K}$ and $1.7 \times 10^{6} \mathrm{~cm}^{-3} \mathrm{~K}$ with $\chi^{2} \sim 10^{-1}$ to $10^{-2}$ over the map ${ }^{3}$ The best $P$ and $G_{\mathrm{UV}}$ maps are represented in Fig. 15. The peaks of $G_{\mathrm{UV}}$ and $P$ are almost cospatial. The maximum is located north of R136, at the southern edge of the [O III] peak. It shows that there is a void around R136 and that the radiation field is first interacting with any matter a few parsecs away from the cluster. Details regarding the structure around R136 are discussed in Sect. 5.3. The uncertainties associated with the observations, including calibration errors, lead to uncertainties on $G_{\mathrm{UV}}$ of $+55 \% /-40 \%$ and on $P$ of $+/-12 \%$.

The value of the observed ratio [OI] $63 \mu \mathrm{m} /[\mathrm{CII}]$ is lower than the ratio predicted by the model based on [C II], [O I] $145 \mu \mathrm{m}$ and $L_{\mathrm{FIR}}^{\mathrm{PDR}}$ by a factor of 1.3-2.5. This discrepancy between the predicted and observed values may be due to optical depth effects of the [OI] $63 \mu \mathrm{m}$ line (Tielens \& Hollenbach 1985; Abel et al. 2007) or to absorption by cold gas along the line of sight (Liseau et al. 2006). As we can see from Fig. 16 on the left, the lowest values of the ratio $\frac{([\mathrm{O} \mathrm{I}] 63 \mu \mathrm{m} /[\mathrm{C} \mathrm{II}])_{\text {observed }}}{([\mathrm{O} \mathrm{I}] 63 \mu \mathrm{m} /[\mathrm{C} \mathrm{II}])_{\text {predicted }}}$ correspond to the locations of the highest pressure. Since the difference between the observations and model prediction seems to correlate spatially with the pressure here, it is probably not due to foreground absorption, but most likely from local effects. We can also note that the observed ratio [O I] $145 \mu \mathrm{m} /[\mathrm{O} \mathrm{I}] 63 \mu \mathrm{m}$ is larger than 0.1 in many pixels, which is an indication of optical depth effects in [O I] $63 \mu \mathrm{m}$ (Tielens \& Hollenbach 1985). In the best-solution model, the predicted ratio [O I] $145 \mu \mathrm{m} /[\mathrm{O}$ I] $63 \mu \mathrm{m}$ is 0.04 . The model accounts for the opacity of the lines for one cloud, but not between several clouds along the line of sight. If we examine one of the best solutions for region $\mathrm{B}$ ( $[\mathrm{C} \mathrm{II}]$ peak in Fig. 3), the model predicts an opacity of 0.6 for [O I] $63 \mu \mathrm{m}$ at $A_{V}=1$ while that of [C II] is still below 0.1 . Thus, if we have several components along the line of sight, the [C II] intensity can be multiplied by the number of components, while the [O I] $63 \mu \mathrm{m}$ intensity increases less than linearly.

\subsection{Determination of $A_{V}^{\max }$ using [C II], [C I] and CO}

In this section we investigate the influence of $A_{V}^{\max }$ (that is, the total depth of the cloud, in magnitude) on the line ratios predicted by the Meudon PDR code. In Fig. 17, we show the ratios $\mathcal{R}$ between several modeled line ratios and their observations for simulated clouds of different total depths. While the ratios [O I] $/[\mathrm{C} \mathrm{II}],([\mathrm{O} \mathrm{I}]+[\mathrm{C} \mathrm{II}]) / L_{\mathrm{FIR}}$ and [C I] $609 \mu \mathrm{m} /[\mathrm{C} \mathrm{I}] 370 \mu \mathrm{m}$ are not very sensitive to $A_{V}^{\max }$ (Fig. 13), the ratios $[\mathrm{C} \mathrm{II}] /[\mathrm{CI}]$ and $[\mathrm{C} \mathrm{II}] / \mathrm{CO}$ vary by several orders of magnitude with the total depth of the simulated cloud because they involve tracers that

\footnotetext{
3 This is not a reduced $\chi^{2}$ as we have as many constraints as free parameters.
} 

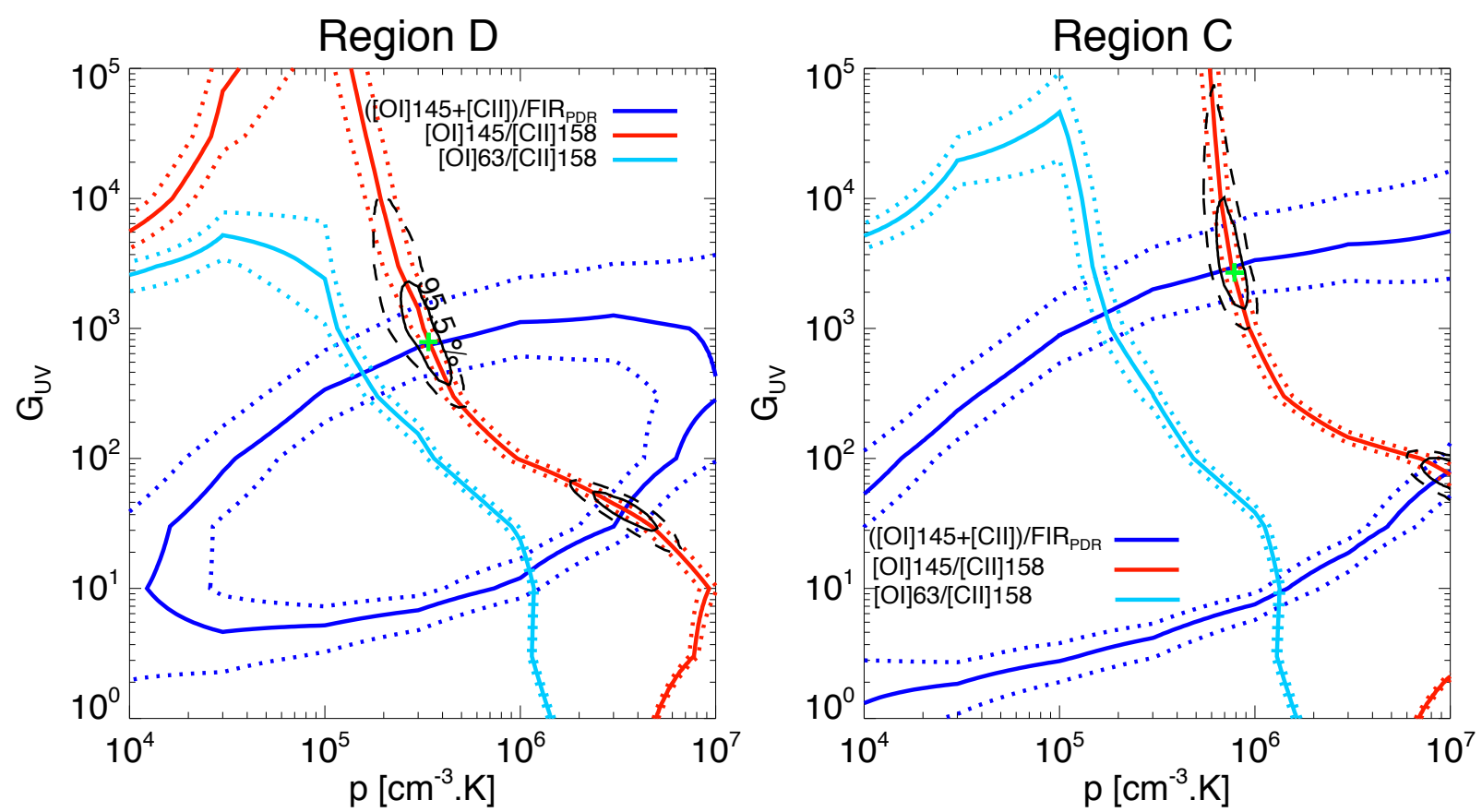

Fig. 14. Contour plots showing the parameters $G_{\mathrm{UV}}$ and $P$ from our model, which reproduces the observed values of the ratios ([O I] $145 \mu \mathrm{m}+[\mathrm{C} \mathrm{II}]) / L_{\mathrm{FIR}}^{\mathrm{PDR}}$ (dark blue), [O I] $145 \mu \mathrm{m} /[\mathrm{C} \mathrm{II}]$ (red), and [O I] $63 \mu \mathrm{m} /[\mathrm{C} \mathrm{II}]$ (cyan). The dotted lines show the $1 \sigma$ error on the observed ratios. The solid and dashed black contours represent the confidence intervals for 1 and $2 \sigma$, respectively. The green crosses indicate the best solutions for $G_{\mathrm{UV}}$ and $P$ in each case, using $([\mathrm{OI}] 145 \mu \mathrm{m}+[\mathrm{C} \mathrm{II}]) / L_{\mathrm{FIR}}^{\mathrm{PDR}}$ and $[\mathrm{OI}] 145 \mu \mathrm{m} /[\mathrm{C} \mathrm{II}]$ as model constraints. Left plot is for a pixel representative of the region D (from Fig. 3). Right plot is for a pixel from region C (from Fig. 3), near the $G_{\mathrm{Uv}}$ peak.
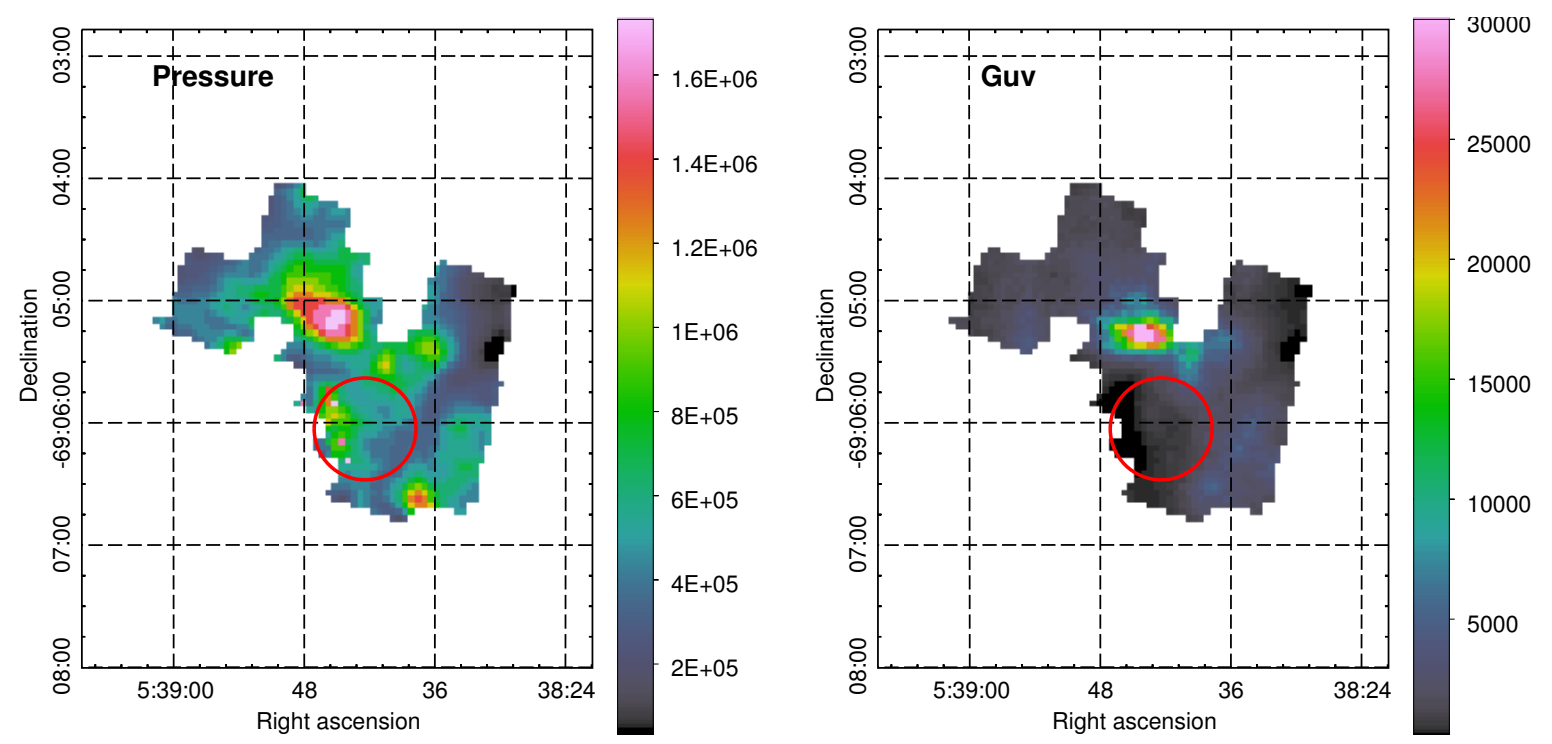

Fig. 15. Left: best pressure map $\left(\mathrm{cm}^{-3} \mathrm{~K}\right)$ of the 30Dor region; using $([\mathrm{O} \mathrm{I}] 145 \mu \mathrm{m}+[\mathrm{C} \mathrm{II}]) / L_{\mathrm{FIR}}^{\mathrm{PDR}}$ and $[\mathrm{OI}] 145 \mu \mathrm{m} /[\mathrm{C}$ II $]$. The total extinction for this model is $A_{V}^{\max }=3$. Right: best $G_{\mathrm{UV}}$ map (color bar in units of the Mathis field) for the same model. The red circle shows the location of R136.

originate from different depths into the cloud. The observed ratios $[\mathrm{C} \mathrm{II}] /[\mathrm{C} \mathrm{I}],[\mathrm{C} \mathrm{II}] / \mathrm{CO}(1-0)$ and $[\mathrm{C} \mathrm{II}] / \mathrm{CO}(3-2)$ can be reproduced with a cloud of $A_{V}^{\max } \sim 1-3 \mathrm{mag}$ per pixel of $30^{\prime \prime}(7.2 \mathrm{pc})$ as shown in Fig. 17 with $G_{\mathrm{UV}}$ and $P$ determined as described in Sect. 4.3. However we keep in mind that the determination of this parameter is strongly dependent on the geometry of the model. This is discussed in more detail in Sect. 5.2.

\subsection{Isobaric versus isochoric case}

We considered a constant density model to compare our results with our isobaric model and with previous PDR model results.
We use the same set of line ratios and the same $\chi^{2}$ method to find the best radiation field and density predicted by an isochoric model.

We find a similar map for $G_{\mathrm{UV}}$ compared to our isobaric model with a maximum of $20 \%$ difference between both cases. For the isochoric model, the density ranges between $3 \times 10^{2}$ to $1.4 \times 10^{4} \mathrm{~cm}^{-3}$ with a spatial distribution that is very similar to $P$ in the isobaric case. This range of values is similar to those found by previous studies (e.g., Poglitsch et al. 1995; Bolatto et al. 1999; Röllig et al. 2006; Pineda et al. 2012). From the results of the isobaric model, we determine the density at the surface of the PDR. This initial density is about $1 / 2$ to $2 / 3$ the 
M. Chevance et al.: A milestone toward understanding PDR properties in the extreme environment of LMC-30 Doradus
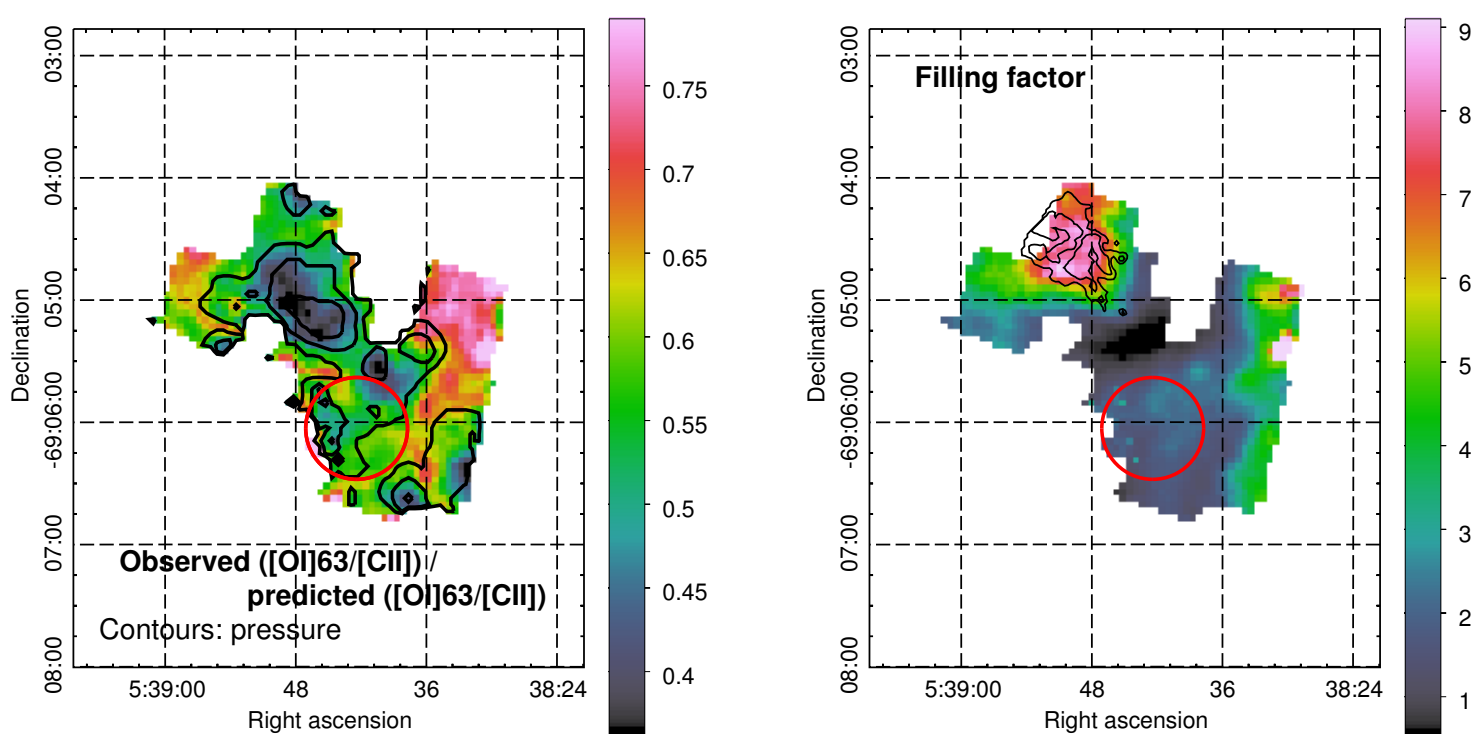

Fig. 16. Left: ratio of the observed [O I] $63 \mu \mathrm{m} /[\mathrm{C}$ II] ratio over the predicted [O I] $63 \mu \mathrm{m} /[\mathrm{C}$ II] ratio (color bar). The pressure contours of between $4.5 \times 10^{5}$ and $1.3 \times 10^{6} \mathrm{~cm}^{-3} \mathrm{~K}$ are indicated in black. The red circle shows the location of R136. Right: filling factor $\Phi_{\mathrm{A}}$ between 0.6 and 9 (color bar). The lowest values are at the peak of $G_{\mathrm{UV}}$. The black contours represent the ${ }^{12} \mathrm{CO}(2-1)$ emission from ALMA (Indebetouw et al. 2013)

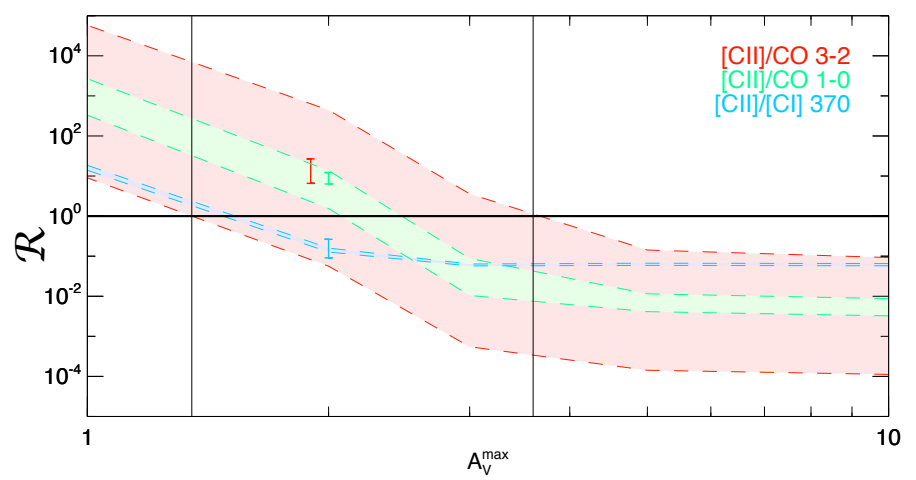

Fig. 17. Same as Fig. 13, for ratios $[\mathrm{C} \mathrm{II}] / \mathrm{CO}(3-2),[\mathrm{C} \mathrm{II}] / \mathrm{CO}(1-0)$ and $[\mathrm{C} \mathrm{II}] /[\mathrm{C} \mathrm{I}] 370 \mu \mathrm{m}$.

density determined with the isochoric model. In conclusion, the results from these two models are similar when applied to PDR line ratios.

However, the predictions of the models start to diverge deeper into the cloud, in particular for molecular lines. Indeed, for an isobaric cloud of $A_{\mathrm{V}}^{\max }$ of 3 , the density rises by about a factor of 10, as the temperature drops, between the surface of the PDR and the core of the cloud (Fig. 12). This implies that the $\mathrm{CO}$ lines are emitted at lower $A_{\mathrm{V}}$ in a isobaric model compared to an isochoric model. As a consequence, the $A_{\mathrm{V}}^{\max }$ probed by the observed $\mathrm{CO}$ lines is lower for an isobaric model than for an isochoric model. For example, the $A_{\mathrm{V}}^{\max }$ probed with the low$J$ CO transitions $(J=3-2$ and $J=1-0)$ is slightly higher for the isochoric model ( 2-4 mag) than the 1-3 mag we find with the isobaric model. In conclusion, choosing the isobaric case is important to reproduce the higher $\mathrm{J}$ transitions of $\mathrm{CO}$ and quantify the CO-dark gas (see Paper II), but it has few consequences for the results derived in this paper.

\subsection{Model predictions for $\mathrm{H}_{2}$ lines}

The $\mathrm{H}_{2} \mathrm{~S}(2)$ line is barely detected in the Spitzer/IRS lowresolution observations of 30Dor (Indebetouw et al. 2009). The model-predicted $\mathrm{H}_{2}(0,0) S(2)$ emission at $12.3 \mu \mathrm{m}$ is 10 to
50 times lower than the upper limit from the observations. However, the emission from the high-resolution spectrum (Sect. 2.4) is in better agreement (by a factor of 2 to 5) with the model prediction.

Pak et al. (1998) measure $\mathrm{H}_{2}(1,0) S(1)$ and $(2,1) S(1)$ close to the $\mathrm{CO}(1-0)$ peak of 30 Dor with a beam of $81^{\prime \prime}$. We compare these observations to the predictions of our model at $81^{\prime \prime}$ resolution. Our map does not fully cover the $81^{\prime \prime}$ beam. However, we calculate lower limits of $6 \times 10^{-6}$ and $3 \times 10^{-6} \mathrm{erg} \mathrm{s}^{-1} \mathrm{~cm}^{-2} \mathrm{sr}^{-1}$ on the $\mathrm{H}_{2}(1,0) S(1)$ and $(2,1) S(1)$ emission, respectively. There is a good agreement with the values measured by Pak et al. (1998) considering we are missing some fraction of their beam $(10.8 \times$ $10^{-6}$ and $4.0 \times 10^{-6} \mathrm{erg} \mathrm{s}^{-1} \mathrm{~cm}^{-2} \mathrm{sr}^{-1}$ respectively). Rubio et al. (1998) present $\mathrm{H}_{2}(1,0) S(1)$ observations at $1.16^{\prime \prime}$ resolution. Their peak value of $4-6 \times 10^{-5} \mathrm{erg} \mathrm{s}^{-1} \mathrm{~cm}^{-2} \mathrm{sr}^{-1}$ is also consistent with our model results.

Yeh et al. (2015) presented an $\mathrm{H}_{2}(1,0) S(1)$ map of the entire 30Dor nebula at $1^{\prime \prime}$ resolution. The peak $\mathrm{H}_{2}$ surface brightness in their region $\mathrm{A}$ is $9.15 \times 10^{-5} \mathrm{erg} \mathrm{s}^{-1} \mathrm{~cm}^{-2} \mathrm{sr}^{-1}$. Our model predicts a maximum $\mathrm{H}_{2}$ surface brightness of $5.3 \times$ $10^{-5} \mathrm{erg} \mathrm{s}^{-1} \mathrm{~cm}^{-2} \mathrm{sr}^{-1}$ located near the [C II] peak for a $12^{\prime \prime}$ resolution. This is about a factor of 2 lower than the observed value, but it may be explained by the lower resolution used in our study and the fact that the $\mathrm{H}_{2}$ emission could originate from clouds smaller than the PACS beam size.

\section{Discussion}

\subsection{Filling factor}

The model assumes that the PDR filling factor is unity, i.e., that the surface area of PDRs is equal to the beam area. If only part of the beam is covered or, on the contrary, if several clouds are present along the line of sight, the radiation emitted in the $L_{\mathrm{FIR}}$ and in cooling lines is different than the model prediction for optically thin lines. The filling factor is, respectively, lower or higher than 1 .

We determined $G_{\mathrm{UV}}$ and $P$ only using ratios of lines coming from the same phase of the ISM. Thus, they do not depend on 
the filling factor because each line is affected by the same factor The area filling factor, $\Phi_{\mathrm{A}}$, can then be estimated from the ratio of the observed intensity over the predicted intensity (for which $\Phi_{\mathrm{A}}=1$ ) for an individual line.

Both $L_{\mathrm{FIR}}^{\mathrm{PDR}}$ and $[\mathrm{CII}]$ are emitted by PDRs and scale with the filling factor in the same way. Thus, we can determine $\Phi_{\mathrm{A}}$ either with $\frac{[\mathrm{C} \text { II }]_{\text {observed }}}{[\mathrm{C} \text { II }]_{\text {predicted }}}$ (as in Wolfire et al. 1990) or similarly with $\frac{L_{\mathrm{FIR} \text { observed }}^{\mathrm{PDR}}}{L_{\mathrm{FIR} \text { predicted }}^{\mathrm{PDR}}}$. This result is presented in the right panel of Fig. 16. The parameter $\Phi_{\mathrm{A}}$ is the smallest (about 0.6) near the peak of $G_{\mathrm{UV}}$ in region C (Fig. 3). The maximum value is $\sim 9$ in the northeast of the cluster. As stated earlier, an area filling factor greater than one means that several clouds covering the entire pixel are along the line of sight. This approach is marginally coherent near the peak of [C II] where the maximum filling factor is $\sim 9$. As noted in Sect. 4.3, considering that the opacity of [C II] is close to 0.1 at the $[\mathrm{C} \mathrm{II}]$ peak, nine clouds on the line of sight add up to an opacity close to 1 . As a consequence, the [C II] intensity does not add up linearly and this may call into question our assumption of optically thin $[\mathrm{C} \mathrm{II}]$ at the $[\mathrm{C} \mathrm{II}]$ peak location. Different PDR viewing angles can also affect this scenario and the number of components we derive is an upper limit.

This spatial distribution of the filling factor reflects the idea that the PDR clouds are smaller and/or less numerous where the radiation field is higher. The region mapped with ALMA by Indebetouw et al. (2013) corresponds to the region where the filling factor of PDRs is the largest in the map (see Fig. 16). The CO emission is highly structured, showing dense $\left(10^{3}-10^{5} \mathrm{~cm}^{-3}\right)$ clumps and filaments, on sub-parsecs scales, covering approximately $10 \%$ of the area of the map. They observed significant photodissociation of $\mathrm{CO}$ by radiation penetrating between the dense clumps, which can explain the increased $X_{\mathrm{CO}}$ factor determined for unresolved low metallicity clouds. Indebetouw et al. measure an $X_{\mathrm{CO}}$ factor that is two times the solar-metallicity value in the dense CO clumps. This value can be even higher, for unresolved studies, when possible interclump $\mathrm{H}_{2}$ is included.

\subsection{Clouds geometry}

Indebetouw et al. (2013) observed the giant molecular cloud 30Dor-10, north of R136 with ALMA during Cycle 0 (it includes our regions $\mathrm{B}$ and $\mathrm{C}$ from Fig. 3). In the region mapped with ALMA, they measure a maximum diameter of $1.2 \mathrm{pc}$ for the $\mathrm{CO}(2-1)$ clumps and a filling factor of about $10 \%$. This could mean that the $\mathrm{CO}$ luminosity is dominated by small, bright clouds in the $\mathrm{CO}$ beam and the medium could be characterized by clumps/filaments of low volume filling factor with a physical size that is much smaller than our pixel size, implying that a plane-parallel geometry together with the high filling factor determined with $[\mathrm{C} \mathrm{II}]$ may not be adapted to our observations.

Indeed, with the $A_{V}^{\max }, G_{\mathrm{UV}}$, and $P$ previously determined in Sects. 4.4 and 4.3, we find that several plane-parallel clouds are required to reproduce the observed absolute intensities. Each of these clouds have a physical size of between 0.2 and $3 \mathrm{pc}$ and an internal $\mathrm{CO}$ layer (estimated where the $\mathrm{CO}$ abundance is at least $50 \%$ of the maximum $\mathrm{CO}$ abundance) that ranges between 0.06 to $0.5 \mathrm{pc}$. The derived size scale is compatible with the ALMA observations. These results however depend on two main assumptions: the plane-parallel geometry and the uniformity of PDR clouds in each pixel.

With a plane geometry, the $A_{V}^{\max }$ of the simulated clouds determined here by comparison of the $[\mathrm{C} \mathrm{II}]$ and $\mathrm{CO}$ emissions can be considered as a lower limit. Indeed, adopting a different geometry by post-processing the results of the model and wrapping the plane-parallel results on a spherical geometry results in a larger $A_{V}^{\max }$. More details about this method can be found in Appendix B. When assuming a spherical geometry, bathed in an isotropic radiation field, a higher $A_{V}(\sim 20 \mathrm{mag}$ at the center of the cloud) is needed to reproduce the $\mathrm{CO}$ observations. Such extinction corresponds to spherical clouds with diameters about four times larger than the size of the optimal plane-parallel clouds. Nevertheless, the results derived in Sect. 4.3 for $G_{\mathrm{UV}}$ and $P$ are independent on the assumed geometry and remain unchanged.

We note that the extinction parameter can be determined independently from the dust mass surface density at a resolution of $22^{\prime \prime}$ (Sect. 2.7). We can then compare $A_{V}^{\text {dust }}$ derived from the dust map to $A_{V}^{\max }$ derived using the $[\mathrm{C} \mathrm{II}] /[\mathrm{C} \mathrm{I}]$ and $[\mathrm{C} \mathrm{II}] / \mathrm{CO}$ ratios in our PDR models (Sect. 4.4). On the one hand, $A_{V}^{\text {dust }}$ corresponds to the integrated extinction along the line of sight in each pixel. On the other hand, $A_{V}^{\max }$ is calculated for individual clouds, so for a meaningful comparison we need to scale by the number of components (i.e., the filling factor $\Phi_{\mathrm{A}}$; Sect. 5.1). While $\Phi_{\mathrm{A}}$ is calculated at a relatively high spatial resolution $\left(12^{\prime \prime}\right)$, the $A_{V}^{\max }$ for individual clouds is not constrained well owing to the poor spatial resolution $\left(42^{\prime \prime}\right)$ and coverage of the $[\mathrm{CI}]$ and $\mathrm{CO}$ maps. In Fig. 18, we plot $A_{V}^{\text {dust }}$ as a function of $\Phi_{\mathrm{A}}$ (recalculated at a resolution of $22^{\prime \prime}$ ) with several tracks showing the scaling with different $A_{V}^{\max }$ values. There is a remarkable correlation between $A_{V}^{\text {dust }}$ and $\Phi_{\mathrm{A}}$, with most data points lying between the $A_{V}^{\max }=$ 1 and 3 lines, in agreement with the values determined with a plane-parallel geometry (Sect. 4.4). We emphasize that the determinations of $A_{V}^{\max }$ and $\Phi_{\mathrm{A}}$ are independent from the determination of $A_{V}^{\text {dust }}$, as the two quantities are derived from different physical processes, and constrained by different observational sets. From Fig. 18, we infer that most clouds share similar $A_{V}^{\max }$ values at a spatial resolution of $22^{\prime \prime}$. Moreover, the good agreement between $A_{V}^{\text {dust }}$ (total extinction) and $A_{V}^{\max }$ (derived in the neutral gas of PDRs) suggests that (1) there is no significant contribution from the ionized gas in the $A_{V}^{\text {dust }}$ determination, and (2) there is no significant contribution from foreground/background gas not associated with 30Dor and not accounted for by our PDR models. Our result not only strengthens the $A_{V}^{\max }$ range obtained in Sect. 4.4, but also suggests that this range remains valid at a resolution of $22^{\prime \prime}$. While this result seems to favor plane-parallel geometry over spherical geometry, we wish to stress that we cannot easily derive the effective extinction corresponding to spherical clouds observed in any given pixel and that the large extinction probed at the center of the spherical cloud is not representative of this effective extinction.

Finally, we discuss the possible existence of clumps embedded in an interclump medium. Since the filling factor determined with [C II] in Sect. 5.1 may characterize an interclump medium surrounding small $\mathrm{CO}$ clumps, it is possible that the [C II] and the $\mathrm{CO}$ emission are not associated with the same structures. For example, a recent study of the N159 region by Okada et al. (2015) find that up to $50 \%$ of [C II] cannot be associated with the $\mathrm{CO}$ emission based on the velocity profiles of the lines. Testing the clumpiness of the medium would require us to adopt a distribution of clouds of different sizes and pressures, illuminated by a central source and including the effect of scattering and shielding, for each pixel of the map. Unfortunately, such a scenario cannot be properly modeled yet because of the lack of observational constraints. 
M. Chevance et al.: A milestone toward understanding PDR properties in the extreme environment of LMC-30 Doradus

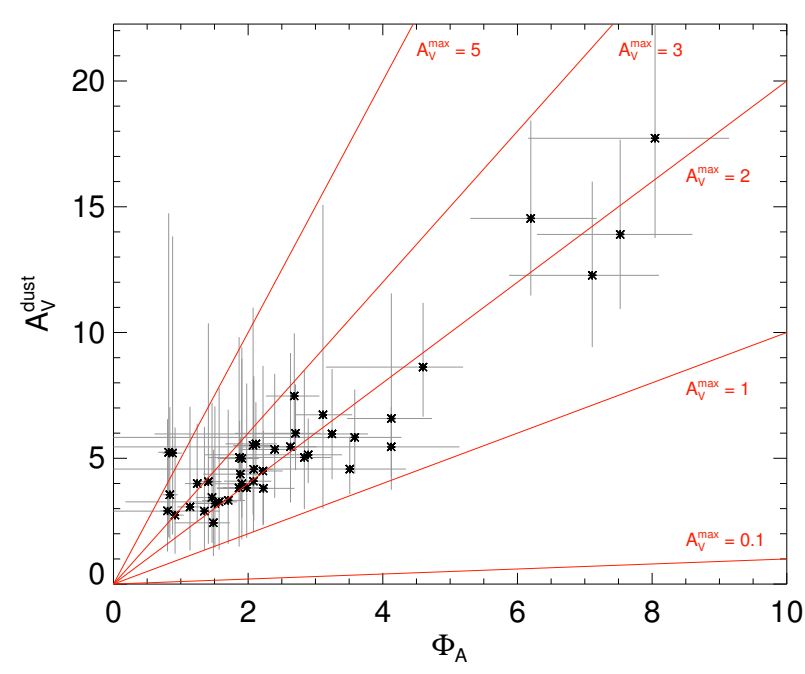

Fig. 18. Extinction derived from the dust mass $A_{V}^{\text {dust }}$ is plotted against the filling factor $\Phi_{\mathrm{A}}$. The diagonal lines show different values of the extinction in individual PDR clouds $\left(A_{V}^{\max }\right)$. The data points agree with $A_{V}^{\text {dust }}$ for $A_{V}^{\max } \sim 1-3$.

\subsection{Three-dimensional distribution of the gas}

In this section, we determine the physical distance between the clouds and ionizing sources to reconstruct the $3 \mathrm{D}$ distribution of the gas. This is accomplished by comparing the incident $G_{\mathrm{UV}}$, predicted by the Meudon PDR code and the emitted radiation field, $G_{\text {stars }}$. We define $G_{\text {stars }}$ as the FUV radiation field computed from the known massive stellar population from the literature. We use catalogs of stars from Crowther \& Dessart (1998) and Selman \& Melnick (1999), including O and B stars and WolfRayet (WF) stars. We use the temperature of the brightest optical sources and integrate over a blackbody between 912 and $2400 \AA$ to be consistent with the definition of $G_{\mathrm{UV}}$ in the Meudon PDR code. We then use a $\frac{1}{R^{2}}$ relation, where $R$ is defined as the physical distance from the center of the cluster, to calculate an average $G_{\text {stars }}$ in each pixel of a cube centered on R136. We first make the assumption that all of the stars lay on the same plane, and derive the $G_{\text {stars }}$ presented in Fig. 19. The maximum incident radiation field we expect on each point of the cube is then $G_{\text {stars }}$, since no absorption is taken into account.

The ratios used to constrain $G_{\mathrm{UV}}$ and $P$ are independent of the filling factor, since [OI] $145 \mu \mathrm{m},\left[\mathrm{C}\right.$ II] and $L_{\mathrm{FIR}}^{\mathrm{PDR}}$ are supposed to be almost cospatial in the PDR model. Thus, $G_{\mathrm{UV}}$ determined with the model is also independent of the filling factor. In that case, the incident radiation field, $G_{\mathrm{UV}}$, should be equal to the emitted radiation field, $G_{\text {stars }}$, modulated by the distance to the R136 plane, assuming there is no absorption between the ionizing stars and the PDR (i.e., the distance to the plane calculated here is an upper limit on the actual distance). We can determine simultaneously four parameters: $G_{\mathrm{UV}}, P, \Phi_{\mathrm{A}}$, and $z$ (the distance to the plane of R136) for each pixel of the map, using the following relations

$$
\begin{aligned}
& L_{\mathrm{FIR} \text { obs }}^{\mathrm{PDR}}=\Phi_{\mathrm{A}} \times L_{\mathrm{FIR} \text { pred }}^{\mathrm{PDR}} \\
& \mathrm{G}_{\mathrm{stars}}=G_{\mathrm{UV}} \times \frac{z^{2}+d^{2}}{d^{2}} \\
& I_{j}=M_{j},
\end{aligned}
$$

where $d$ is the projected distance between a pixel and the central source, $\sqrt{z^{2}+d^{2}}=R, I_{j}$ are the observed values of the line

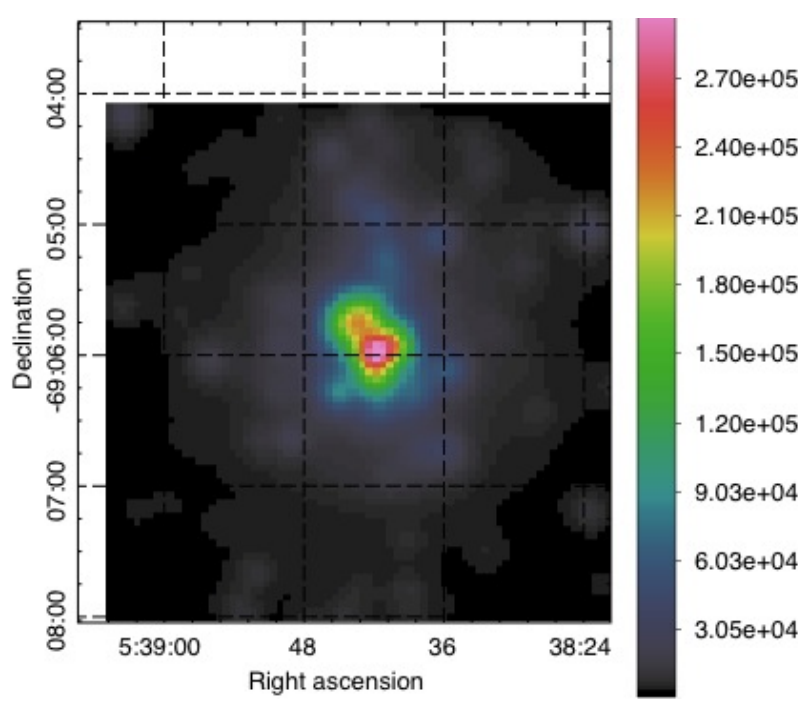

Fig. 19. Intensity of the emitted radiation field $G_{\text {stars }}$ in the plane of R136 at $12^{\prime \prime}$ resolution (color bar in units of the Mathis field).

intensity ratios $j$, and $M_{j}$ the ratios calculated by the PDR model (in erg s${ }^{-1} \mathrm{~cm}^{-2} \mathrm{sr}^{-1}$ ). The parameters $G_{\mathrm{UV}}$ and $G_{\text {stars }}$ are in units of the Mathis field, and $G_{\mathrm{UV}}$ and $P$ are calculated as described in Sect. 4.3, using Eq. (4).

The physical distance $R$ between PDR clouds and R136 is presented in Fig. 20. It ranges from $\sim 11$ to $80 \mathrm{pc}$. The gas located close to R136 in the projected view is actually 40 to 80 pc away from the cluster. The bright arm-like structure in [O III], which, when projected, appears further from R136, is much closer to the star cluster as it is almost on the same plane. This is consistent with the distance calculated in Pellegrini et al. (2011) for the ionized gas using optical observations.

The physical distance described above was derived by assuming that all of the stars are located in the same plane. For comparison, we also considered a random distribution of the stars in the perpendicular direction, but maintaining a high density of stars within a 6 pc radius around the center of R136 in order to reproduce a spherical distribution. We performed a Monte Carlo simulation and calculated distances similar to our previous determination ( $<40 \%$ difference throughout the map). This is not surprising as most stars in R136 are in fact located in $\mathrm{a} \sim 6 \mathrm{pc}$ radius sphere. We used the Monte Carlo simulation to estimate a typical uncertainty on the physical distance of $\sim 4 \mathrm{pc}$.

\subsection{Porosity of the ISM}

The [O III] $88 \mu \mathrm{m}$ line emission is detected over large spatial scales in 30Dor, as has already been noticed in other extended sources (e.g., in N11 by Lebouteiller et al. 2012) and, together with the small-scale CO clumps we see, may be indicative of a highly porous region. There is probably mixing of the ionized and neutral phases of the ISM throughout all spatial scales. This idea is supported by Fig. 21, which shows the ratio [O III]/[C II] as a function of $\Phi_{\mathrm{A}}$. We can see that these two quantities are strongly correlated. Indeed, a low area filling factor $\Phi_{\mathrm{A}}$ in a given pixel implies a small volume fraction occupied by PDRs. This means that there is less matter per unit volume to absorb the UV radiation and this radiation is able to travel further away. Region B from Fig. 3 (violet), at $\sim 40$ pc from R136, has the lowest $[\mathrm{O}$ III $] /[\mathrm{C}$ II $]$ ratio and the highest PDR filling factor. This is also where the $[\mathrm{C} \mathrm{II}]$ peaks and the $\mathrm{CO}$ clumps reside (see contours in 


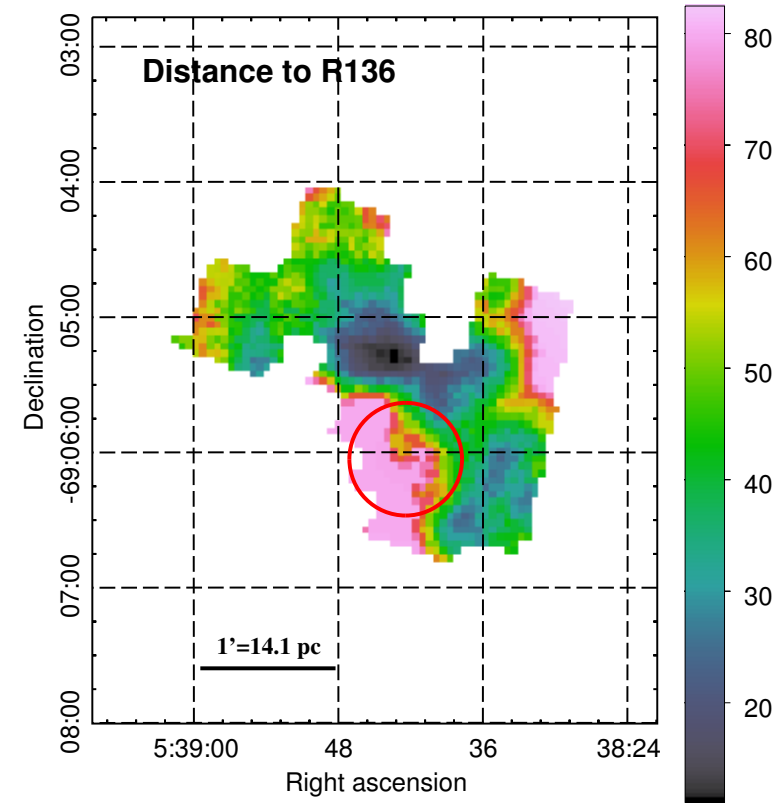

Fig. 20. Physical distance $R$ (in pc) of the surface of the PDR to the center of R136. The red circle shows the location of R136.

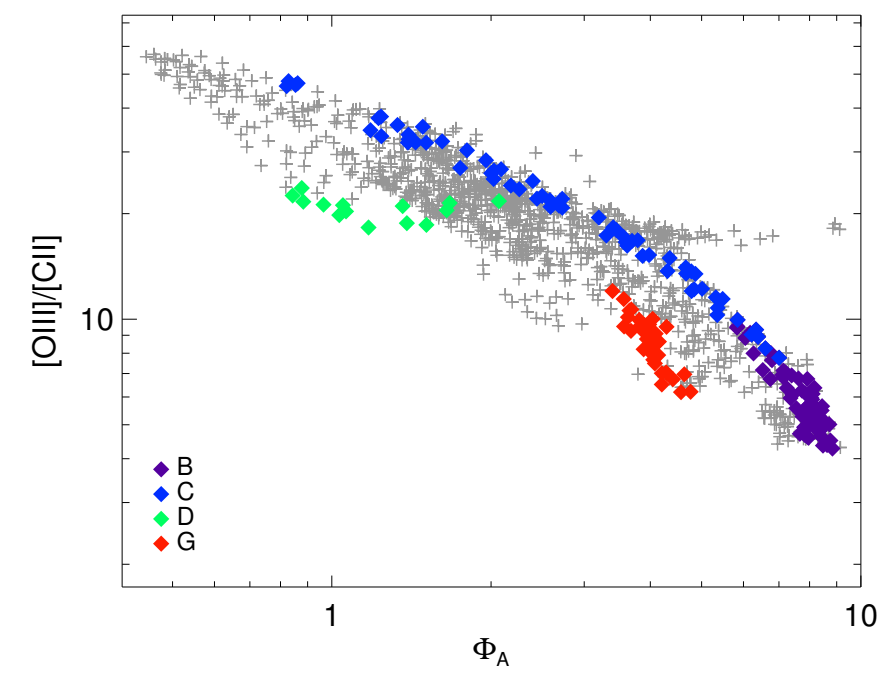

Fig. 21. Correlation between $[\mathrm{O} I I I] /[\mathrm{C} \mathrm{II}]$ and the PDR area filling factor. The color symbols are associated with the regions defined in Fig. 3. The gray symbols are all of the other pixels of our 30Dor map.

Fig. 16). In contrast, the ratio $[\mathrm{O} \mathrm{III}] /[\mathrm{C} \mathrm{II}]$ is high $(>10)$ in region $\mathrm{D}$ (green), which is one of the furthest region from R136 with a low $\Phi_{\mathrm{A}}$. The blue points of region $\mathrm{C}$, which is the [O III] peak and near the $G_{\mathrm{UV}}$ and $P$ peaks, show a large range of $[\mathrm{O} \mathrm{III}] /[\mathrm{C} \mathrm{II}]$ and $\Phi_{\mathrm{A}}$. This is a region of widely varying ISM conditions. The ratio $[\mathrm{O} \mathrm{III}] /[\mathrm{C} \mathrm{II}]$ as a function of the physical distance $\mathrm{R}$ or $G_{\mathrm{UV}}$ is a scatter plot, highlighting the fact that the proximity of the ionizing source is not the only controlling factor of the structure of the ISM.

Over the mapped area of $42 \mathrm{pc} \times 56 \mathrm{pc}$ the PDR filling factor and the $[\mathrm{O} \mathrm{III}] /[\mathrm{C} \mathrm{II}]$ ratio vary over one order of magnitude. The decrease in dust abundance and intense UV photons from the SSC conspire together to shape the surrounding porous ISM, filling it with hard photons and a relatively small filling factor of PDR clumps.

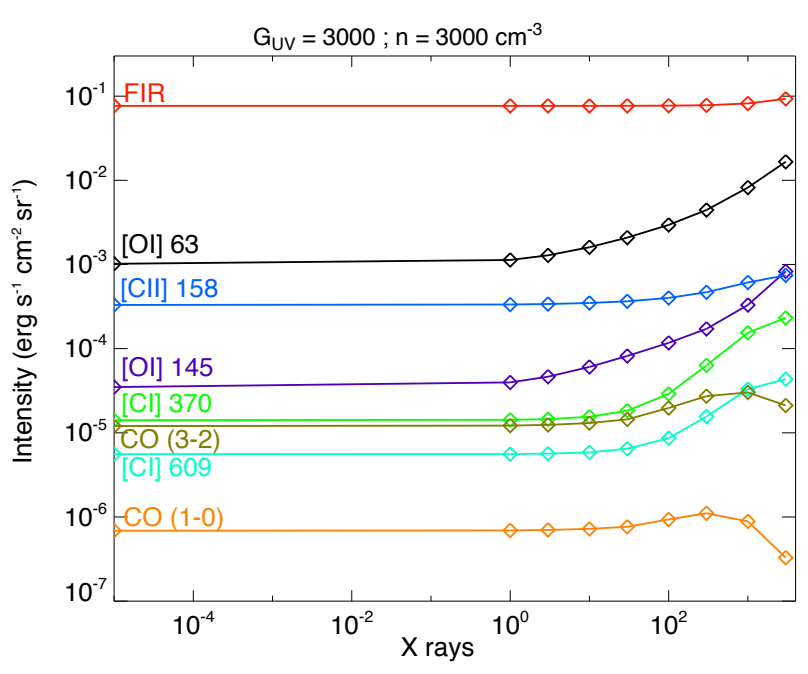

Fig. 22. Influence of the X-ray luminosity on the intensities of individual lines for a typical isochoric model with $G_{\mathrm{UV}}=3 \times 10^{3}$ and $n=3 \times 10^{3} \mathrm{~cm}^{-3} . G_{X}$ is the X-ray luminosity integrated between 0.2 and $8 \mathrm{keV}$, given in unit of the Habing field (Habing 1968). This is to be compared with our results for $G_{\mathrm{UV}}$ (see Sect. 5.5).

\subsection{Other sources of excitation}

No evidence of any shock tracers has been found in 30Dor by previous studies so far (Indebetouw et al. 2009; Yeh et al. 2015). We investigate here the possibility for X-rays to be an important source of excitation of the gas in 30Dor. Townsley et al. (2006) studied the population of X-ray point sources (energy between 0.5 and $8 \mathrm{keV}$ ) in a $17^{\prime} \times 17^{\prime}$ field around R136 with Chandra. Spectral fitting is performed on the brightest sources (49 in total). In particular, they determine a total X-ray luminosity, corrected for absorption, of $10^{36.95} \mathrm{erg} \mathrm{s}^{-1}$ for the brightest source in R136 (Mk 34) with an X-ray flux of $2 \times 10^{-3} \mathrm{erg} \mathrm{s}^{-1} \mathrm{~cm}^{-2} \mathrm{sr}^{-1}$ at a distance of $20 \mathrm{pc}$ away, approximately at the ionization front. As presented in Fig. 22, such a low $G_{\mathrm{X}}$, compared to the values of $G_{\mathrm{UV}}$, does not have an important effect on the intensity of the individual lines for a given $G_{\mathrm{UV}}$ and density.

\subsection{If 30Dor were unresolved}

Studying resolved nearby galaxies can help us understand more distant unresolved targets. At a distance of $900 \mathrm{kpc}$, the entire region of 30Dor mapped in [O I] $145 \mu \mathrm{m}$ with PACS (covering $56 \mathrm{pc} \times 70 \mathrm{pc}$ ) would fall in only one spaxel of the PACS spectrometer. This distance is comparable to the distance of the Andromeda galaxy $(\sim 780 \mathrm{kpc})$.

We integrate all of the tracers we have and perform the same study at this lower resolution with only one pixel. Using the same technique as Sect. 4.3, we find that all of the [C II] emission originates from the PDRs. Using the Meudon PDR code, we determine $G_{\mathrm{UV}}=1390, P=6.18 \times 10^{5} \mathrm{~cm}^{-3} \mathrm{~K}$, and $A_{V}=2-3$. This is representative of a region of moderate $G_{\mathrm{UV}}$ and $P$ in our detailed spatial study, such as region $\mathrm{G}$ in Fig. 3 or the ISM north of region $B$.

Even though our map is relatively small and centered on $\mathrm{R} 136$, the global solution is already biased toward the solution corresponding to regions with relatively low $P$ and low $G_{\mathrm{UV}}$. However, we have to keep in mind that the regions targeted with PACS are the most luminous around R136, especially for the [O I] $145 \mu \mathrm{m}$ line. This implies that if we were to integrate an even larger area, the result would be presumably biased even 
more to this diffuse and low $G_{\mathrm{UV}}$ regime. These two parameters could be even lower if we include more diffuse regions, which is the subject of a subsequent study in the LMC.

\section{Conclusions}

We have studied the ISM properties in the extreme environment of 30Dor in the LMC, around the super star cluster R136. We summarize our results as follow:

1. We presented and analyzed new Herschel PACS observations of 30Dor in [C II] $158 \mu \mathrm{m}$, [O I] 63 and $145 \mu \mathrm{m}$, [N II] 122 and $205 \mu \mathrm{m}$, and [O III] $88 \mu \mathrm{m}$ over a $56 \times 70$ pc region of 30 Dor. All of these lines are well detected and provide diagnostics on the structure of the ISM.

The [O III] line is the brightest of the FIR lines, ranging from 2 to 60 times more luminous than [C II]. We propose that the [O I] $63 \mu \mathrm{m}$ line is optically thick throughout the mapped region. We find that the $[\mathrm{C} \mathrm{II}] / \mathrm{CO}(1-0)$ luminosity ranges between $5 \times 10^{4}$ and $3 \times 10^{5}$ throughout the map. This range is larger than the broad range of ratios found from integrated DGS dwarf galaxies of Cormier et al. (2014).

2. Using the Spitzer and Herschel MIR to submm photometric observations, we model the full dust SED spatially around 30Dor and derive the infrared luminosity map. We find that the [C II] intensity ranges from 0.1 to $1 \%$ of the observed $L_{\mathrm{FIR}}$ throughout the region mapped.

3. Based on the electron density of the ionized gas (10 to $100 \mathrm{~cm}^{-3}$ ) and the high value of the $[\mathrm{C} \mathrm{III}] /[\mathrm{N} \mathrm{II}]$ ratio, we determined that at least $90 \%$ of the $[\mathrm{C}$ II $]$ emission originates from the PDRs, which makes the [C II] intensity a valuable constraint for our PDR modeling.

4. We decomposed the $L_{\text {FIR }}$ map to separate the component associated with the ionized gas from the PDR-only component. We find, in places, that $\sim 70 \%$ of the $L_{\mathrm{FIR}}$ is not from PDRs, but associated with the ionized gas component, which we remove for the PDR modeling. We emphasize caution when applying the total $L_{\mathrm{FIR}}$ to PDR models without considering the origin of the $L_{\text {FIR }}$.

5. From the ratios ([O I $] 145 \mu \mathrm{m}+[\mathrm{C}$ II $]) / L_{\mathrm{FIR}}^{\mathrm{PDR}}$ and [OI] $145 \mu \mathrm{m} /[\mathrm{C}$ II $]$ we determined the spatial distribution of the radiation field and the pressure with the Meudon PDR code. The parameter $G_{\mathrm{UV}}$ ranges between $10^{2}$ and $3 \times 10^{4}$ (in units of the standard radiation field defined in Mathis et al. 1983) and $P$ ranges between $10^{5}$ and $1.7 \times 10^{6} \mathrm{~cm}^{-3} \mathrm{~K}$.

6. The total depth of the clouds is determined by including the ratios $[\mathrm{C} \mathrm{II}] /[\mathrm{C} \mathrm{I}]$ or $[\mathrm{C} \mathrm{II}] / \mathrm{CO}$ in the modeling assuming that all of these tracers are associated with the same structures. We showed that in the 30Dor region, $A_{V}^{\max }$ in $30^{\prime \prime}$ pixels is $\sim 1-3 \mathrm{mag}$. This value should be considered a minimum value because of our assumption of a plane-parallel geometry.

7. We conducted a 3D model of the PDR gas around R136. A comparison of the incident radiation field determined from our PDR model, $G_{\mathrm{UV}}$, with the emitted radiation field, $G_{\text {stars }}$, reveals that the PDR gas is distributed at various distances, ranging between 20 to $80 \mathrm{pc}$ from the excitation source, R136.

8. The PDR area filling factor ranges between 0.6 (at the peak of $G_{\mathrm{UV}}$ ) and 9 (at the peak of [C II]). The high value of the $[\mathrm{O} \mathrm{III}] /[\mathrm{C} \mathrm{II}]$ ratio and its tight correlation with the filling factor rather than with the distance, highlight the porosity of the
ISM, filled with hard photons around relatively small PDR clumps.

The combined effects of a half-solar metallicity gas with the intense excitation source R136 create the extreme environment we see in 30Dor. It has been shown that the structure of the gas in this region is dominated by photoionization. X-rays or shocks are not needed to reproduce the observed line intensities. Based on our findings in the present study, we speculate that the small size of the $\mathrm{CO}$ core inside the PDR clouds could explain the high $[\mathrm{C} \mathrm{II}] / \mathrm{CO}$ ratio we observe in low metallicity environments. The high value of the [O III] emission line suggests that a highly porous medium is a characteristic of the gas in low metallicity dwarf galaxies.

Acknowledgements. The authors would like to thank Annie Hughes for providing the $\mathrm{CO}(1-0)$ data and Akiko Kawamura for the $\mathrm{CO}(3-2)$ data. This research was made possible through the financial support of the Agence Nationale de la Recherche (ANR) through the programme SYMPATICO (Program Blanc Projet (NR-11-BS56-0023) and also through the EU FP7. SH acknowledges financial support from DFG programme HO 5475/2-1. PACS was developed by MPE (Germany); UVIE (Austria); KU Leuven, CSL, IMEC (Belgium); CEA, LAM (France); MPIA (Germany); INAFIFSI/OAA/OAP/OAT, LENS, SISSA (Italy); IAC (Spain). This development was supported by BMVIT (Austria), ESA-PRODEX (Belgium), CEA/CNES (France), DLR (Germany), ASI/INAF (Italy), and CICYT/MCYT (Spain). SPIRE was developed by Cardiff University (UK); Univ. Lethbridge (Canada); NAOC (China); CEA, LAM (France); IFSI, Univ. Padua (Italy); IAC (Spain); SNSB (Sweden); Imperial College London, RAL, UCL-MSSL, UKATC, Univ. Sussex (UK) and Caltech, JPL, NHSC, Univ. Colorado (USA). This development was supported by CSA (Canada); NAOC (China); CEA, CNES, CNRS (France); ASI (Italy); MCINN (Spain); Stockholm Observatory (Sweden); STFC (UK); and NASA (USA).

\section{References}

Abel, N. P., Sarma, A. P., Troland, T. H., \& Ferland, G. J. 2007, ApJ, 662, 1024 Allen, M. G., Groves, B. A., Dopita, M. A., Sutherland, R. S., \& Kewley, L. J. 2008, ApJS, 178, 20

Aniano, G., Draine, B. T., Gordon, K., \& Sandstrom, K. M. 2011, PASP, 123, 1218

Asplund, M., Grevesse, N., Sauval, A. J., \& Scott, P. 2009, ARA\&A, 47, 481

Bernard-Salas, J., Habart, E., Arab, H., et al. 2012, A\&A, 538, A37

Bolatto, A. D., Jackson, J. M., \& Ingalls, J. G. 1999, ApJ, 513, 275

Bolatto, A. D., Wolfire, M., \& Leroy, A. K. 2013, ARA\&A, 51, 207

Brauher, J. R., Dale, D. A., \& Helou, G. 2008, ApJS, 178, 280

Bron, E., Le Bourlot, J., \& Le Petit, F. 2014, A\&A, 569, A100

Cigan, P., Young, L., Cormier, D., et al. 2016, AJ, 151, 14

Cormier, D., Madden, S. C., Hony, S., et al. 2010, A\&A, 518, L57

Cormier, D., Lebouteiller, V., Madden, S. C., et al. 2012, A\&A, 548, A23

Cormier, D., Madden, S. C., Lebouteiller, V., et al. 2014, A\&A, 564, A121

Cormier, D., Madden, S. C., Lebouteiller, V., et al. 2015, A\&A, 578, A53

Crowther, P. A., \& Dessart, L. 1998, MNRAS, 296, 622

Fazio, G. G., Hora, J. L., Allen, L. E., et al. 2004, ApJS, 154, 10

Fulton, T. R., Baluteau, J.-P., Bendo, G., et al. 2010, Proc. SPIE, 7731, 773134

Galliano, F., Hony, S., Bernard, J.-P., et al. 2011, A\&A, 536, A88

Gordon, K. D., Engelbracht, C. W., Rieke, G. H., et al. 2008, ApJ, 682, 336

Habing, H. J. 1968, Bull. Astron. Inst. Netherlands, 19, 421

Heyminck, S., Graf, U. U., Güsten, R., et al. 2012, A\&A, 542, L1

Hunter, D. A. 1999, in New Views of the Magellanic Clouds, eds. Y.-H. Chu,

N. Suntzeff, J. Hesser, \& D. Bohlender, IAU Symp., 190, 217

Hunter, D. A., Kaufman, M., Hollenbach, D. J., et al. 2001, ApJ, 553, 121

Indebetouw, R., de Messières, G. E., Madden, S. C., et al. 2009, ApJ, 694, 32

Indebetouw, R., Brogan, C., Chen, C. H. R., et al. 2013, ApJ, 774, 73

Indriolo, N., \& McCall, B. J. 2012, ApJ, 745, 91

Indriolo, N., Neufeld, D. A., Gerin, M., et al. 2015, ApJ, 800, 40

Kaufman, M. J., Wolfire, M. G., Hollenbach, D. J., \& Luhman, M. L. 1999, ApJ, 527,795

Langer, W. D., Velusamy, T., Pineda, J. L., Willacy, K., \& Goldsmith, P. F. 2014, A\&A, 561, A122

Le Bourlot, J., Le Petit, F., Pinto, C., Roueff, E., \& Roy, F. 2012, A\&A, 541, A76 Le Petit, F., Nehme, C., Le Bourlot, J., \& Roueff, E. 2006, ApJS, 164, 506 Lebouteiller, V., Bernard-Salas, J., Brandl, B., et al. 2008, ApJ, 680, 398 Lebouteiller, V., Cormier, D., Madden, S. C., et al. 2012, A\&A, 548, A91 Liseau, R., Justtanont, K., \& Tielens, A. G. G. M. 2006, A\&A, 446, 561 
Madden, S. C., Poglitsch, A., Geis, N., Stacey, G. J., \& Townes, C. H. 1997, ApJ, 483, 200

Madden, S. C., Rémy-Ruyer, A., Galametz, M., et al. 2013, PASP, 125, 600

Mathis, J. S., Mezger, P. G., \& Panagia, N. 1983, A\&A, 128, 212

Meixner, M., Gordon, K. D., Indebetouw, R., et al. 2006, AJ, 132, 2268

Meixner, M., Panuzzo, P., Roman-Duval, J., et al. 2013, AJ, 146, 62

Minamidani, T., Tanaka, T., Mizuno, Y., et al. 2011, AJ, 141, 73

Negishi, T., Onaka, T., Chan, K.-W., \& Roellig, T. L. 2001, A\&A, 375, 566

Oberst, T. E., Parshley, S. C., Nikola, T., et al. 2011, ApJ, 739, 100

Okada, Y., Requena-Torres, M. A., Güsten, R., et al. 2015, A\&A, 580, A54

Ott, S. 2010, Astronomical Data Analysis Software and Systems XIX, 434, 139

Pagel, B. E. J. 2003, in CNO in the Universe, eds. C. Charbonnel, D. Schaerer, \& G. Meynet, ASP Conf. Ser., 304, 187

Pak, S., Jaffe, D. T., van Dishoeck, E. F., Johansson, L. E. B., \& Booth, R. S. 1998, ApJ, 498, 735

Pellegrini, E. W., Baldwin, J. a., \& Ferland, G. J. 2011, ApJ, 738, 54

Pilbratt, G. L., Riedinger, J. R., Passvogel, T., et al. 2010, A\&A, 518, L1

Pineda, J. L., Mizuno, N., \& Röllig, M. 2012, A\&A, 544, A84

Pineda, J. L., Langer, W. D., \& Goldsmith, P. F. 2014, A\&A, 570, A121

Poglitsch, A., Krabbe, A., Madden, S. C., et al. 1995, ApJ, 454, 293

Poglitsch, A., Waelkens, C., Geis, N., et al. 2010, A\&A, 518, L2

Rémy-Ruyer, A., Madden, S. C., Galliano, F., et al. 2013, A\&A, 557, A95

Rémy-Ruyer, A., Madden, S. C., Galliano, F., et al. 2014, A\&A, 563, A31

Rémy-Ruyer, A., Madden, S. C., Galliano, F., et al. 2015, A\&A, 582, A121
Rieke, G. H., Young, E. T., Engelbracht, C. W., et al. 2004, ApJS, 154, 25 Rolleston, W. R. J., Trundle, C., \& Dufton, P. L. 2002, A\&A, 396, 53 Röllig, M., Ossenkopf, V., Jeyakumar, S., Stutzki, J., \& Sternberg, A. 2006, A\&A, 451, 917

Rubio, M., Garay, G., \& Probst, R. 1998, The Messenger, 93, 38

Schruba, A., Leroy, A. K., Walter, F., et al. 2012, AJ, 143, 138

Selman, F., \& Melnick, J. 1999, A\&A, 549, 532

Smith, J. D. T., Armus, L., Dale, D. A., et al. 2007a, PASP, 119, 1133

Smith, J. D. T., Draine, B. T., Dale, D. A., et al. 2007b, ApJ, 656, 770

Stacey, G. J., Geis, N., \& Genzel, R. 1991, ApJ, 373, 423

Stacey, G. J., Hailey-Dunsheath, S., Ferkinhoff, C., et al. 2010, ApJ, 724, 957

Swinyard, B. M., Polehampton, E. T., Hopwood, R., et al. 2013, MNRAS, 440, 3658

Tielens, a. G. G. M., \& Hollenbach, D. 1985, ApJ, 291, 722

Townsley, L. K., Broos, P. S., Feigelson, E. D., et al. 2006, AJ, 131, 2140

Walker, A. R. 2012, Astrophys. Space Sci., 341, 43

Werner, M. W. W., Roellig, T. L. L., Low, F. J. J., et al. 2004, ApJS, 154, 1

Wolfire, M. G., Hollenbach, D., \& McKee, C. F. 2010, ApJ, 716, 1191

Wolfire, M. G., Tielens, A. G. G. M., \& Hollenbach, D. J. 1990, ApJ, 358, 116

Wong, T., Hughes, A., Ott, J., et al. 2011, ApJS, 197, 16

Wu, R., Polehampton, E. T., Etxaluze, M., et al. 2013, A\&A, 556, A116

Wu, R., Madden, S. C., Galliano, F., et al. 2015, A\&A, 575, A88

Yeh, S. C. C., Seaquist, E. R., Matzner, C. D., \& Pellegrini, E. W. 2015, ApJ, 807,117 


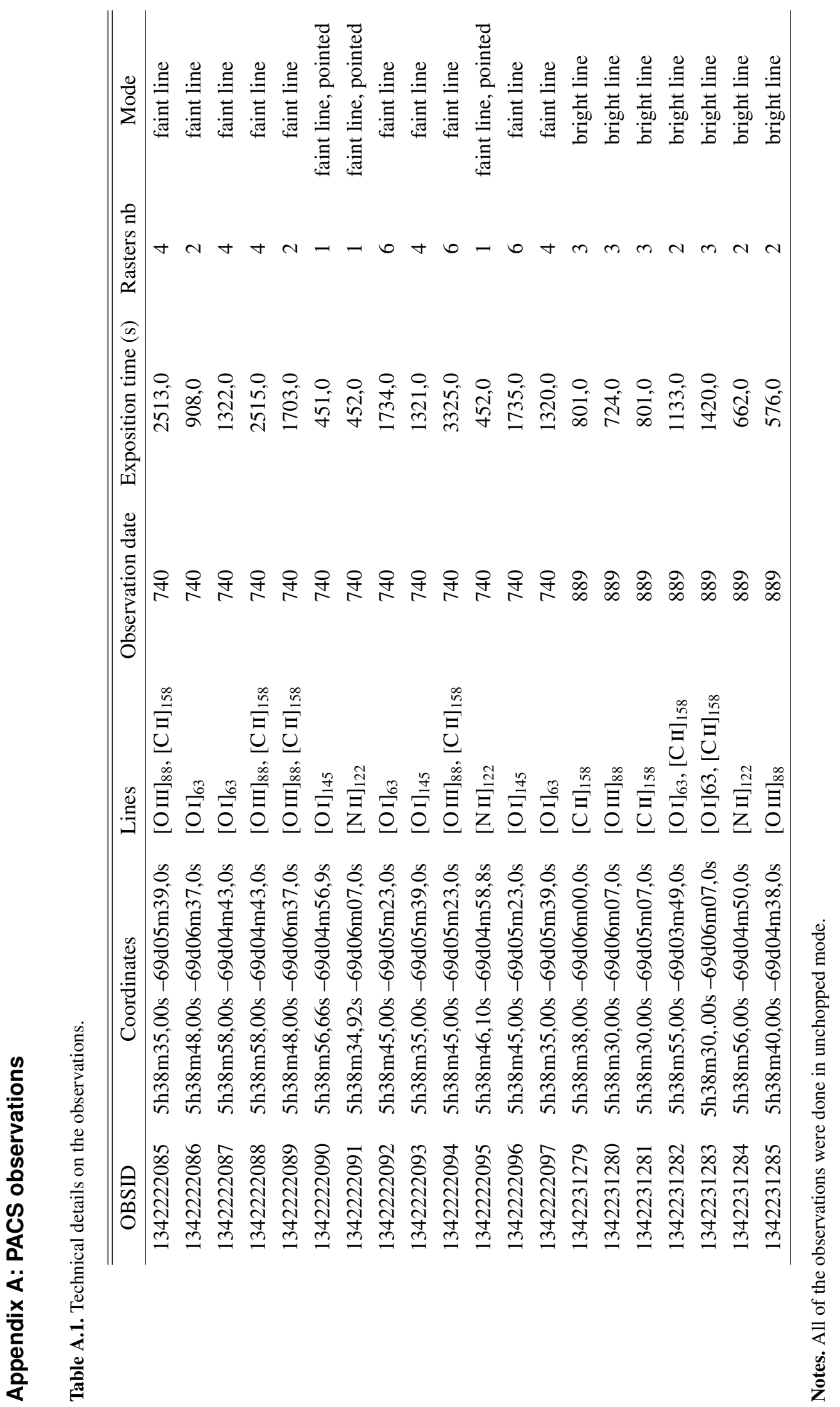




\section{Appendix B: Spherical geometry}
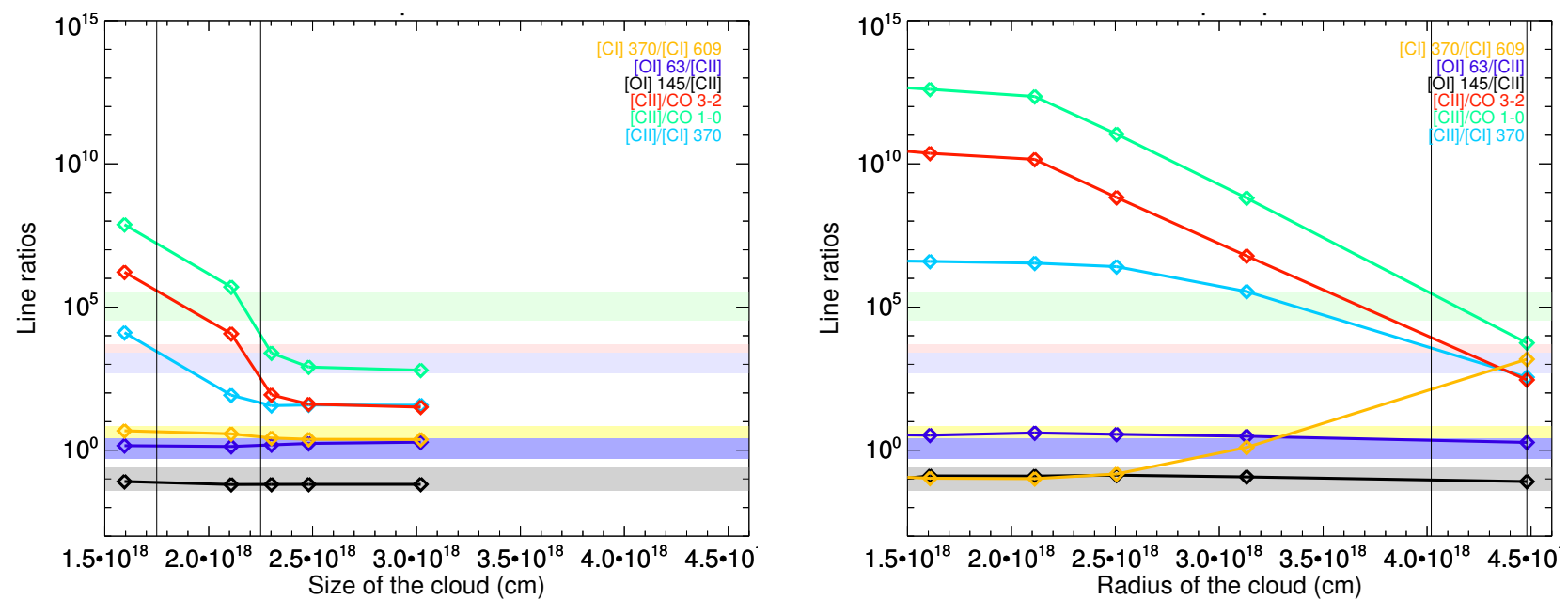

Fig. B.1. Left: ratios $\mathcal{R}$ of the modeled line ratios for [C I] $370 \mu \mathrm{m} /[\mathrm{C}$ I] $609 \mu \mathrm{m}$, [O I] $63 \mu \mathrm{m} /[\mathrm{C} \mathrm{II}]$, [O I] $145 \mu \mathrm{m} /[\mathrm{C} \mathrm{II}]$, [C II]/CO(3-2), [C II]/CO(10 ), and $[\mathrm{C} \mathrm{II}] /[\mathrm{CI}] 370 \mu \mathrm{m}$, for simulated plane-parallel clouds of different sizes with $G_{\mathrm{UV}}=1 \times 10^{3}$ and $P=3 \times 10^{5} \mathrm{~cm}^{-3} \mathrm{~K}$. Right: same for spherical clouds of different radius. The vertical lines indicate the range of radius where the predictions of the model are compatible with the observed $[\mathrm{C}$ II $] / \mathrm{CO}(3-2)$ and $\left[\mathrm{C}_{\mathrm{II}}\right] /[\mathrm{C} \mathrm{I}] 370 \mu \mathrm{m}$.

The Meudon PDR code is a plane-parallel model. The code computes the abundance profiles of the various species and excited states in a plane-parallel system as a function of the distance to the surface of the cloud. It is possible to post-process the results of a simulation to wrap the structure and simulate a spherical cloud. To do that, we integrate the intensity of each transition over a sphere, where the abundance profiles of each species as a function of the distance to the surface of the sphere is equal to the computed abundance profile as a function of the distance to the surface for a plane-parallel geometry. The resulting line ratios as a function of the diameter of the sphere, for an integrated cloud illuminated by an isotropic field, are shown in the right panel of Fig. B.1.
This is approach is geometrical. It is not done to accurately model the physics of a spherical cloud, but to investigate the impact of the geometry on the integrated intensity, similar to the approach or Bolatto et al. (1999). Physically, this approach is only valid when there is enough extinction, i.e., for values of $A_{V} \gtrsim 5$. In addition, we wish to emphasize that the approach of computing a spherical cloud by wrapping the structure is not satisfactory in the case of clouds illuminated by a central stellar cluster since the radiation field seen by any cloud is not isotropic. The ideal model would be a model with clouds of various sizes located at various distances from a central radiation source. 